\title{
Towards models of gravitational waveforms from generic binaries: II. Modelling precession effects with a single effective precession parameter
}

\author{
Patricia Schmidt, ${ }^{1,2,3}$ Frank Ohme, ${ }^{1}$ and Mark Hannam ${ }^{1}$ \\ ${ }^{1}$ School of Physics and Astronomy, Cardiff University, Queens Building, \\ CF24 3AA Cardiff, United Kingdom \\ ${ }^{2}$ LIGO Laboratory, California Institute of Technology, MS 100-36, Pasadena, California 91125, USA \\ ${ }^{3}$ Theoretical Astrophysics 350-17, California Institute of Technology, Pasadena, California 91125, USA
} (Received 26 August 2014; published 29 January 2015)

\begin{abstract}
Gravitational waves (GWs) emitted by generic black-hole binaries show a rich structure that directly reflects the complex dynamics introduced by the precession of the orbital plane, which poses a real challenge to the development of generic waveform models. Recent progress in modelling these signals relies on an approximate decoupling between the nonprecessing secular inspiral and a precession-induced rotation. However, the latter depends in general on all physical parameters of the binary which makes modelling efforts as well as understanding parameter-estimation prospects prohibitively complex. Here we show that the dominant precession effects can be captured by a reduced set of spin parameters. Specifically, we introduce a single effective precession spin parameter, $\chi_{p}$, which is defined from the spin components that lie in the orbital plane at some (arbitrary) instant during the inspiral. We test the efficacy of this parameter by considering binary inspiral configurations specified by the physical parameters of a corresponding nonprecessing-binary configuration (total mass, mass ratio, and spin components (anti) parallel to the orbital angular momentum), plus the effective precession spin applied to the larger black hole. We show that for an overwhelming majority of random precessing configurations, the precession dynamics during the inspiral are well approximated by our equivalent configurations. Our results suggest that in the comparable-mass regime waveform models with only three spin parameters faithfully represent generic waveforms, which has practical implications for the prospects of $\mathrm{GW}$ searches, parameter estimation and the numerical exploration of the precessing-binary parameter space.
\end{abstract}

DOI: 10.1103/PhysRevD.91.024043

PACS numbers: 04.20.Ex, 04.25.Nx, 04.30.Db, 95.30.Sf

\section{INTRODUCTION}

The commissioning of the advanced interferometric gravitational-wave $(\mathrm{GW})$ detectors is currently underway, with Advanced LIGO (aLIGO) [1,2] expected to go online in late 2015, and to reach its anticipated design sensitivity by 2019 [3]. Advanced Virgo [4] in Italy and Kagra [5] in Japan are expected to follow soon after. The coalescence of two compact objects like black holes or neutron stars is among the most promising candidates for the first direct detection of GWs. However, the prime detection strategy for GWs from coalescing compact binaries exploited by the ground-based detectors, matched filtering, relies on theoretical knowledge of the gravitational waveforms. It is therefore crucial to have accurate and efficient waveform models of the GW signal from binary coalescences readily available to use in the advanced-detector era.

The dynamical evolution of a compact binary system can be separated into three distinct stages: the inspiral, the merger and the ringdown, if the final object is a black hole. While inspiral waveforms can be predicted accurately by analytic approximation methods like post-Newtonian (PN) expansions (see Ref. [6] and references therein) or their resummation into effective-one-body (EOB) models [7-9], the later stages need to be calculated from numerical relativity (NR) solutions of the full nonlinear Einstein field equations. Current waveform models for the complete inspiral-merger-ringdown (IMR) GW signal were constructed by combining analytic PN/EOB and NR results.

To date, a number of such theoretical IMR waveform models exist for nonspinning black-hole binaries [10-18] as well as for spinning binary configurations where the orbital angular momentum of the binary motion is (anti) parallel to the spin angular momenta of the individual holes [19-22] (see also Ref. [23] for an overview). In these configurations the black holes orbit in a spatially fixed twodimensional plane, and the dominant mode of the GW signal can be described by simple monotonic functions for the amplitude and phase. The spins modify the inspiral rate and the GW amplitude, but otherwise the GW signals are qualitatively similar between nonspinning and aligned-spin configurations. The NR simulations needed to calibrate these IMR models cover a three-dimensional parameter space of the binary's mass ratio and the two spin magnitudes (the total mass is a simple scaling factor for vacuum solutions). The models produced to date were calibrated with $\sim 30$ NR simulations, cf. Refs. [19,21] and Ref. [24].

In the most general cases, however, the individual spin angular momenta have arbitrary orientations, and any misalignment between the orbital angular momentum 
and the spins causes the orbital plane as well as the spin vectors to precess $[25,26]$. The simple inspiral motion becomes more complicated, leading to a GW signal with amplitude and phase modulations that depend on the orbital-plane orientation, as well as a richer mode structure. We will discuss in more detail the phenomenology of precessing binaries in Sec. II.

During the last four years, a number of key results have helped to develop a simple framework to model the waveforms of precessing black-hole binaries [27-31]. In particular, in earlier work we showed that the waveform from the inspiral of a precessing binary can be approximated by an underlying nonprecessing-binary waveform that has been "twisted up" by the precessional motion of the orbital plane [30] (see Fig. 5 of Ref. [30]). The nonprecessing waveform is characterized by the individual masses and the components of the black-hole spins parallel to the orbital angular momentum, which remain roughly constant throughout the inspiral. Our results indicated that this mapping holds up to the merger; later work quantified that the merger and ringdown can also be mapped to nonprecessing-binary waveforms, but the parameter identification is less clear [31]. The inspiral dynamics (predominantly influenced by the individual masses and the "parallel" spin components) can be approximately decoupled from the precession, which is determined by all of the physical parameters, and this suggests an elegant way to construct a generic-binary model, i.e., to separately produce a waveform model for aligned-spin binaries and an additional model for the precessional motion [30]. This proposal has since been exploited to construct precessing IMR models $[32,33]$.

An open problem in modelling generic binaries (i.e., including precession effects in the merger and ringdown) is the need for NR simulations across a seven-dimensional parameter space (mass ratio, plus the vector components of each black hole's spin). NR simulations are computationally expensive, and even a coarse sampling of four points in each direction of the parameter space would require $4^{7} \sim O\left(10^{4}\right)$ simulations. One way to make this problem tractable is to identify the physical parameters (or combinations of them) that most strongly affect the GW signal. This approach will not only provide us with a smaller subspace over which to perform NR simulations, but will also indicate those physical parameters that can most accurately be measured in future GW observations.

This approach has already been used in some models of spinning, nonprecessing binaries: the spins predominantly affect the inspiral rate, but this influence can be parametrized by a weighted sum of the two spins, and therefore efficient aligned-spin models can be produced with only one spin parameter rather than two [19,21,34-36]. Our goal in this work is to identify a complementary spin parameter for precession and reduce the remaining four dimensions (the in-plane spin components) to a subspace that accurately captures the dominant precession-induced features in GW signals across the full parameter space. We find that a single additional "precession spin parameter," which we denote by $\chi_{p}$, is sufficient for this purpose, and we investigate its efficacy in a study of PN inspiral waveforms for generic comparable-mass-ratio binaries.

Preliminary work on this effective precession spin parameter motivated the choice of parameters in our phenomenological frequency-domain IMR model, PhenomP [33]. This work also provides additional justification for singlespin waveform models, such as the physical template family [37] and the precessing stationary-phase inspiral model in Ref. [38].

This paper is organized as follows. In Sec. II we briefly summarize the phenomenology of simply precessing binaries and recent developments in modelling precessing binaries. In Sec. III we introduce the effective precession spin parameter $\chi_{p}$. In Sec. IV we describe the PN waveforms and analysis procedure we use to quantify the accuracy of waveforms where the in-plane spins are mapped to $\chi_{p}$, and the results are presented in Sec. V. Based on these we discuss the applicability of the $\chi_{p}$ approximation in Sec. VII.

\section{PRECESSING BLACK-HOLE BINARIES}

\section{A. Notation}

Generic binary black holes are in general characterized by seven intrinsic physical parameters: the mass ratio $q=m_{2} / m_{1} \geq 1$, where $m_{i}$ denote the component masses, and the six spin components of their two spin angular momenta $\vec{S}_{i}(i=1,2)$, or their dimensionless counterparts $\vec{\chi}_{i}=\vec{S}_{i} / m_{i}^{2}$. The total mass $M=m_{1}+m_{2}$ of the binary sets the overall scale in general relativity and therefore need not be explicitly included in a waveform model. We denote unit directions by ${ }^{\wedge}$ and lengths by capital letters. The total spin of the binary is given by $\vec{S}=\vec{S}_{1}+\vec{S}_{2}$.

\section{B. Phenomenology}

We briefly summarize the essential features of precession and its effects on the GW signal. For a more detailed discussion we refer the reader to Refs. $[25,26]$.

The loss of binding energy via GWs causes two spinning black holes with component masses $m_{1}$ and $m_{2}$ in a quasicircular orbit to spiral inwards until they merge into a single black hole. If the black holes' spin angular momenta $\vec{S}_{i}$ are aligned (anti)parallel to the orbital angular momentum $\vec{L}$, then the orbital motion occurs in a fixed two-dimensional plane, defined by $\hat{L}$, which is also the direction of dominant GW energy emission.

This simple picture changes when the individual spins have some arbitrary orientation. In such generic configurations, the orientations of the individual spins and the orbital plane evolve. In most configurations the binary follows simple precession, where both the spin and orbital 
angular momenta precess around the binary's total angular momentum, $\vec{J}=\vec{L}+\vec{S}_{1}+\vec{S}_{2}$ [25]. The direction of the total angular momentum is approximately fixed, i.e., $\hat{J}(t) \simeq \hat{J}_{t \rightarrow-\infty}$, and is therefore a natural generalization of the orbital angular momentum as a characteristic direction in the binary system. If $\hat{N}$ is the line-of-sight direction of a distant inertial observer (detector), then we can define $\theta=\measuredangle(\hat{J}, \hat{N})$ as the inclination of the binary.

When $L \simeq S$ and $\hat{L} \sim-\hat{S}$, then small changes in $J$ due to GW emission are comparable to the magnitude of $J$, and its direction is not fixed; on the contrary it "tumbles over" (see Fig. 6 in Ref. [30]). This is called transitional precession. Only a very restricted set of physical configurations will undergo transitional precession while emitting GWs at frequencies within the sensitivity band of the advanced GW detectors, and therefore observations of these systems are expected to be rare [25].

In the following, we adopt a Cartesian coordinate system attached to the binary such that at the initial time $\hat{J}_{0} \equiv \hat{z}$, which we refer to as the $J_{0}$-aligned source frame. Therein, we define the instantaneous direction of the orbital angular momentum, $\hat{L}(t)$, by the two polar angles $(l(t), \alpha(t))$. These functions encode the time evolution of the orientation of the orbital plane in the source frame. The precession cone opening angle $l(t)$ is defined by

$$
l(t):=\arccos (\hat{L}(t) \cdot \hat{J}(t))
$$

and the azimuthal angle $\alpha(t)$ is given by

$$
\alpha(t):=\arctan \left(\frac{L_{y}}{L_{x}}\right) .
$$

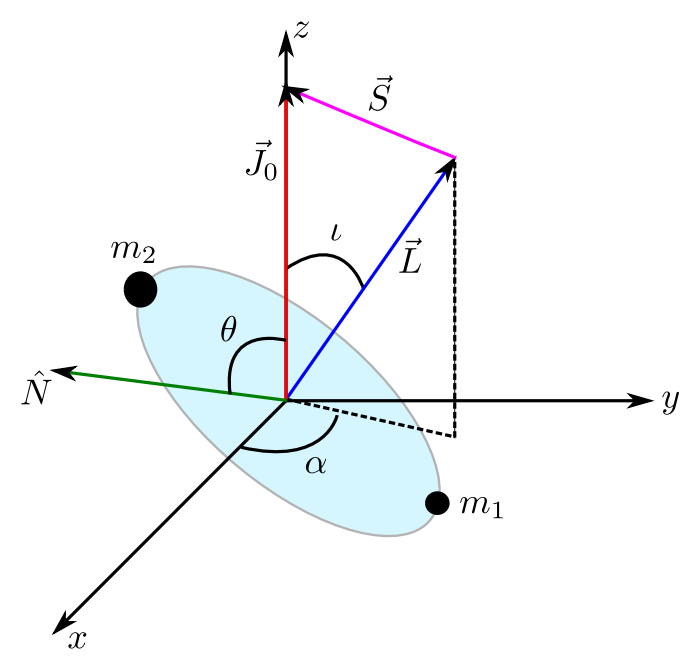

FIG. 1 (color online). The $\hat{J}_{0}$-aligned source frame of a precessing binary. $\theta$ denotes the angle between the line of sight (radiation propagation direction) and the total angular momentum; $\vec{S}=\vec{S}_{1}+\vec{S}_{2}$ is the total spin.
The geometry of a precessing configuration is depicted in Fig. 1. Due to its nature, the azimuth angle is directly related to the precession frequency, i.e., the rate at which $\hat{L}$ precesses around $\hat{J}$,

$$
\omega_{p}(t)=\frac{d \alpha(t)}{d t} .
$$

Precession occurs due to spin-orbit and spin-spin couplings and is a purely relativistic effect. In PN theory, the evolution of $L$ and $S_{i}$ is described at leading order in spinorbit, spin-spin and radiation-reaction contributions by the precession equations given in Eqs. (A1)-(A3). The dominant effect occurs at 1.5-PN order (spin-orbit coupling) and drives the precession of the orbital plane (LenseThirring precession); the dominant spin-spin coupling term appears at 2PN order and induces nutational motion. The precession of the orbital plane and the spins alter the otherwise simple orbital motion and consequently affect the GW emission. Most importantly, precession introduces a secular modification to the signal phase $\Phi(t)$, given by

$$
\Phi(t)=\int_{0}^{t}\left(\omega_{\mathrm{orb}}\left(t^{\prime}\right)-\dot{\alpha}\left(t^{\prime}\right) \cos \imath\left(t^{\prime}\right)\right) d t^{\prime}
$$

as well as amplitude and phase modulations, and in the relative amplitudes of the waveform modes. We emphasize that the strength of the modulations depends strongly on the relative orientation of the binary to the observer, i.e., $\theta$. Even strongly precessing systems can show only mild modulations if the observer is aligned with $\hat{J}_{0}$, i.e., $\theta=0$. The effect of the orientation on the modulations is illustrated in Fig. 2.

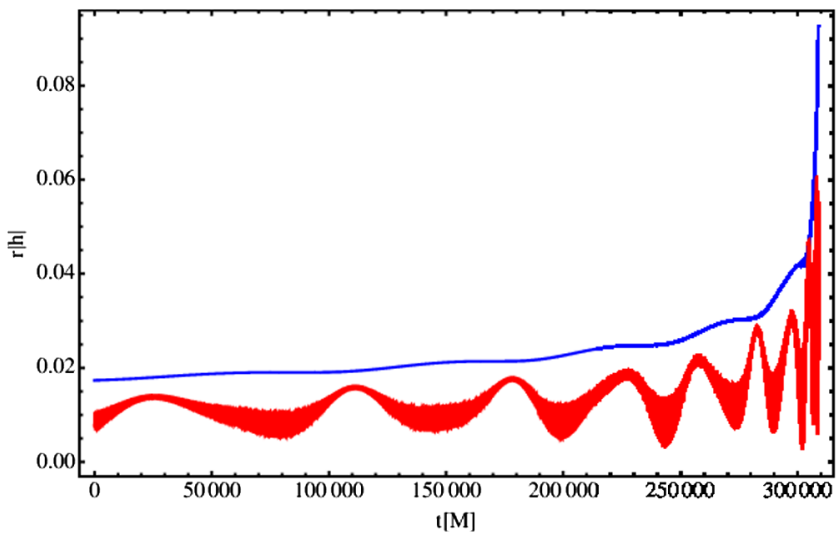

FIG. 2 (color online). Magnitude of the GW strain $h$ computed with all $l=2$ modes for a precessing binary, where the total angular momentum $\hat{J}_{0}$ is aligned with the line of sight (top blue curve) and for the arbitrary orientation $(\theta, \phi)=\left(60^{\circ}, 113^{\circ}\right)$ (lower red curve). The binary's parameters are $q=3$, $\vec{\chi}_{1}=(1,0,0)$ and $\vec{\chi}_{2}=(0.8,0,0.6)$. While only weak amplitude modulations are visible along $\hat{J}_{0}$, we observe strong modulations for the arbitrary orientations. 


\section{Modelling simple precession: summary and recent progress}

Since accurate waveform templates are a key ingredient in most detection and parameter-estimation strategies, finding accurate and efficient waveform models for generic binaries has been an ongoing challenge for several decades. In the inspiral regime this has meant finding simple ways to capture the dominant precession effects, without having to solve the full PN or EOB equations of motion, which is prohibitively expensive in GW applications. Here we give a brief overview of the most important recent developments but refer to Ref. [39] for a more complete treatment.

First attempts to construct search templates for precessing signals [40,41] followed soon after the analysis of the phenomenology of precessing binaries within the PN framework by Apostolatos et al. [25] and Kidder [26]. Apostolatos was the first to observe the potential of modulating the secular phase, which he referred to as the "carrier phase," to describe the total phase of the precessing system. Schematically, the precessing GW strain $h$ is then given as

$$
h(t)=\Lambda(t) h_{C}(t),
$$

where $h_{C}(t)$ is the unmodulated carrier signal and $\Lambda(t)$ is a complex factor containing all information regarding the precession-induced modulations of the amplitude and the phase [see Eqs. (6)-(17) in Ref. [40] for details]. Crucially, this ansatz assumes that the unmodulated carrier phase is that of a nonspinning binary. Apostolatos concluded that the agreement between the artificially modulated waveforms and true precessing waveforms is unacceptably low even for moderate precession [40].

Subsequently, Buonanno, Chen and Vallisneri [42] modified the modulation factor in Apostolatos' general ansatz. However, the description of the secular phase was unchanged. The modified $\Lambda(t)$ was able to capture the precession-induced modulations better, but in order to do so, up to six free nonphysical parameters were introduced, which were subsequently shown to admit waveforms that mimicked detector noise and lead to an increase in the false-alarm rate of a GW search [43].

In previous work [30], we have suggested modelling GWs from generic black-hole binaries in a similar way, but we identified the carrier signal with an appropriate aligned-spin waveform which is "twisted up" following the precession dynamics. We proposed

$$
\begin{aligned}
h_{C}^{\text {nonspinning }}(t) & \rightarrow h^{\text {nonprecessing }}(t), \\
\Lambda(t) & \rightarrow \mathbf{R}(t),
\end{aligned}
$$

where the modulation factor $\Lambda$ becomes a simple rotation operator $\mathbf{R}$ with a concrete physical meaning: it encodes the evolution of the orbital plane.
While aligned-spin binaries have been accurately modelled in the past, the missing ingredient is a sufficient description of the rotation operator $\mathbf{R}$, which depends on the precession angles $l$ and $\alpha$. Exact solutions to the leading-order PN precession equations are known for two special cases, equal-mass or single-spin binaries [25], but in general analytic solutions are not known in the comparablemass regime. In addition, the angles $\alpha$ and $l$ depend in general on all six spin components, which significantly complicates modelling efforts. In order to establish a sufficiently accurate but simple model for the two angle functions, it would be advantageous to reduce the number of dependent parameters, and we shall motivate a single parameter that governs the precession dynamics in the following section.

\section{EFFECTIVE PRECESSION SPIN}

In previous work [30,31], it was shown that the secular phasing, i.e., the inspiral rate, of precessing binaries is determined by the mass ratio and spin components parallel to the orbital angular momentum, $S_{i \|}=\vec{S}_{i} \cdot \hat{L}$. These are approximately constant, in that they exhibit only small variations throughout the inspiral, even for generic binaries, and the oscillations always have an amplitude of $\Delta \chi_{i}<0.1$. This behavior is illustrated in Fig. 3 for a precessing binary with mass ratio $q=3, \vec{\chi}_{1}=(0.4,-0.2,0.3)$ and $\vec{\chi}_{2}=(0.75,0.4,-0.1)$. (The details of our PN waveform generation are given in Appendix A.) We see that the parallel spin components each oscillate around a mean value, which is close to the initial values of $S_{1 \|}=0.01875$ and $S_{2 \|}=-0.05625$. Note that the individual total spin magnitudes $S_{i}$ are conserved, and the observed oscillations in the parallel spin magnitudes are compensated by changes in the in-plane spin magnitudes at each moment in time which in turn is illustrated in Fig. 4. We note that these

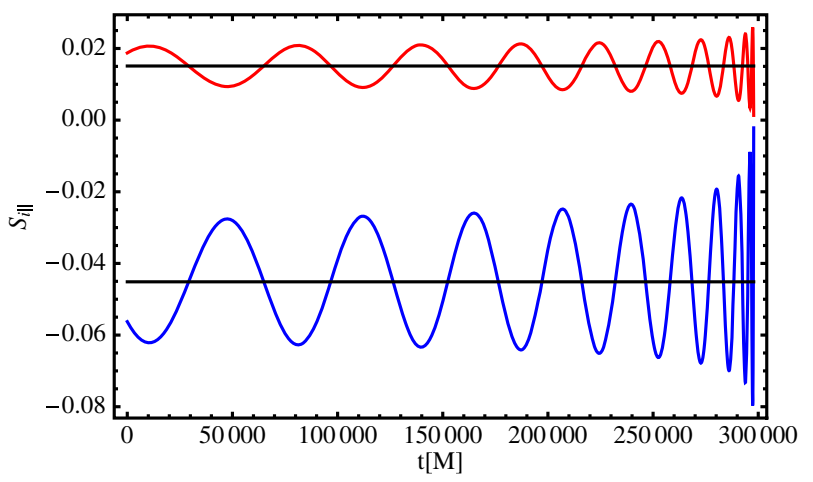

FIG. 3 (color online). Evolution of the two spins parallel to the orbital angular momentum. The top red graph shows the evolution of the parallel spin of the smaller black hole, $S_{1 \|}$, and the lower blue curve that of the parallel spin of the larger black hole, $S_{2 \|}$ for the case described in the text. The two horizontal lines indicate the mean value of each parallel spin with $\bar{S}_{1 \|}=0.015$ and $\bar{S}_{2 \|}=-0.045$. 

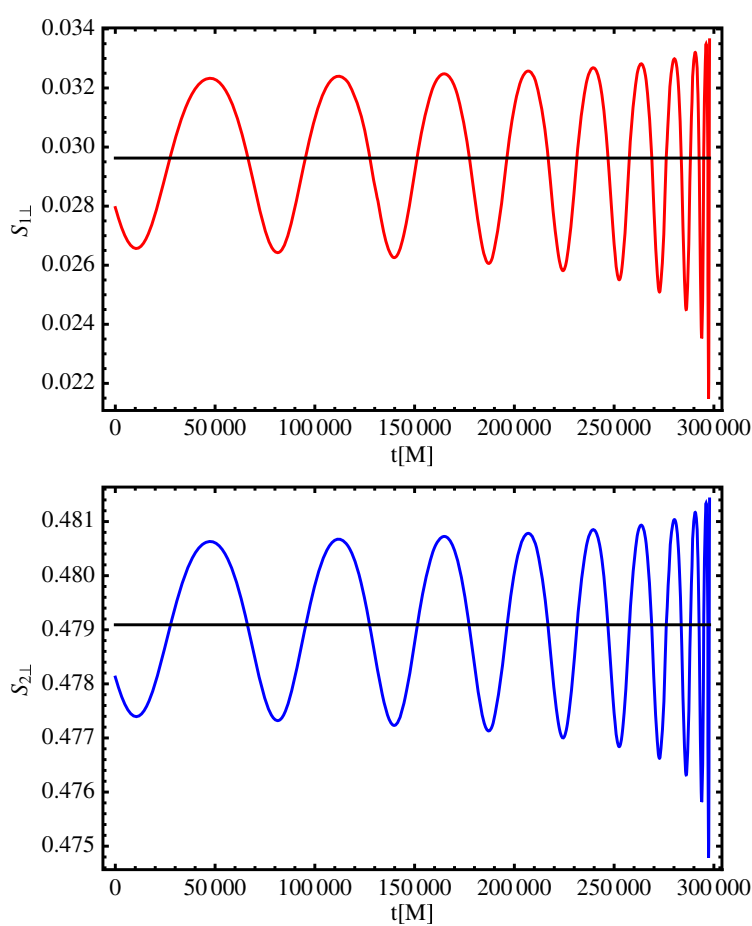

FIG. 4 (color online). The top panel shows the evolution of $S_{1 \perp}$ as a function of time, and the bottom panel shows the evolution of $S_{2 \perp}$. Similar to the parallel spin magnitudes, the in-plane spin magnitudes oscillate around some mean values, which are $\bar{S}_{1 \perp}=0.030$ and $\bar{S}_{2 \perp}=0.479$, respectively (horizontal lines).

oscillations occur on the precession and not the orbital time scale, and, once again, the in-plane spin magnitudes oscillate around approximately fixed mean values. For comparison, in this case the initial in-plane magnitudes were 0.0279 and 0.478 .

To describe the precession, we require additional information from the spin components that lie in the orbital plane orthogonal to $\hat{L}$. It is therefore convenient to decompose the spin vectors with respect to $\hat{L}$ into their parallel and orthogonal vector components such that each spin vector $\vec{S}_{i}=\vec{S}_{i \|}+\vec{S}_{i \perp}$. In the following, however, we will show that it is possible to faithfully approximate the precession in a generic binary system by combining these four in-plane spin components $\vec{S}_{1 \perp}$ and $\vec{S}_{2 \perp}$ into only one additional spin parameter, a complementary effective precession spin, $\chi_{p}$.

Consider the leading-order PN precession equation [25]:

$$
\begin{aligned}
\dot{\vec{L}} & =\frac{L}{r^{3}}\left[\left(2+\frac{3 q}{2}\right) \vec{S}_{1}+\left(2+\frac{3}{2 q}\right) \vec{S}_{2}\right] \times \hat{L} \\
& \equiv \frac{L}{r^{3}}\left[A_{1} \vec{S}_{1 \perp}+A_{2} \vec{S}_{2 \perp}\right] \times \hat{L},
\end{aligned}
$$

where $A_{1}=2+3 q / 2$ and $A_{2}=2+3 /(2 q)$, and $r$ denotes the separation. We see immediately that the in-plane spins
$\vec{S}_{i \perp}$ drive the evolution of $L$. Similar evolution equations are given for the spin vectors [see Eqs. (A2)-(A3)]. At leading order these suggest that the in-plane spins $\vec{S}_{i \perp}$ rotate within the orbital plane, but with different rotational velocities, i.e., they have different precession rates around $\hat{L}$. Their magnitudes $S_{i \perp}$ may also oscillate, as shown in Fig. 4, indicating the nutation of the orbital plane. The magnitude of these oscillations is typically small, and need not be modeled accurately in order to describe the waveform faithfully (as quantified in Sec. IV). Instead, in the following we focus on modelling the average precession of the orbital plane.

The two observations we have just made, 1) that the magnitudes of the in-plane spins $S_{i \perp}$ each oscillate around a mean value and 2) that the relative angle between the spin vectors in the plane changes continuously, suggest a simple way to construct a single precession spin parameter. At some times during the inspiral, the two in-plane spin vectors will be parallel, and will add together in Eq. (3.2). At other times, the in-plane spin vectors will point in opposite directions, and their contributions will be minimized. Over many precession cycles, the overall contribution to Eq. (3.2) can be approximated by the average magnitude of these two contributions:

$$
\begin{aligned}
S_{p} & :=\frac{1}{2}\left(A_{1} S_{1 \perp}+A_{2} S_{2 \perp}+\left|A_{1} S_{1 \perp}-A_{2} S_{2 \perp}\right|\right) \\
& \equiv \max \left(A_{1} S_{1 \perp}, A_{2} S_{2 \perp}\right) .
\end{aligned}
$$

This parameter can be defined at any point during the inspiral, and the variation from the true mean value will typically be small. This is illustrated in Fig. 5. We see that $S_{p}$ is directly related to the in-plane spin angular momentum of one of the black holes. As we will see below, in most configurations this $i s$ the in-plane spin of the larger black hole.

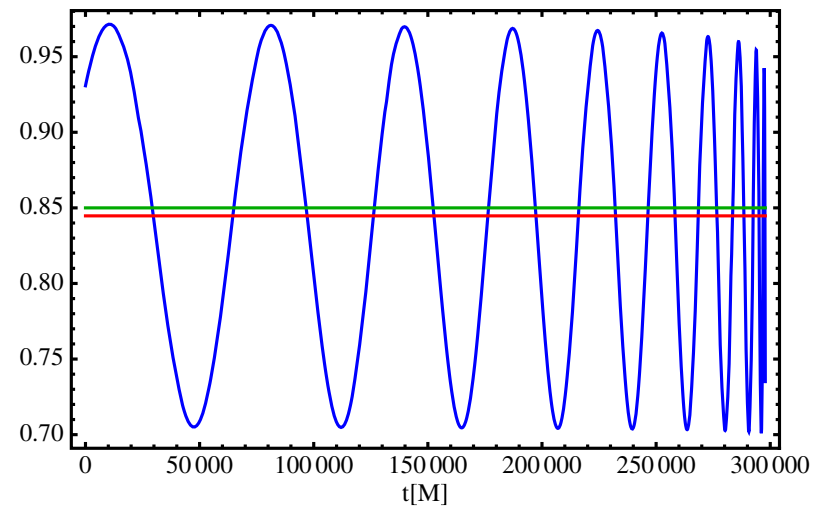

FIG. 5 (color online). Magnitude of the leading-order precession term $\left\|\left(A_{1} \vec{S}_{1}+A_{2} \vec{S}_{2}\right) \times \hat{L}\right\| /\left(A_{2} m_{2}^{2}\right)$ (blue), its true mean $\left\|\left(A_{1} \vec{S}_{1}+A_{2} \vec{S}_{2}\right) \times \hat{L}\right\| /\left(A_{2} m_{2}^{2}\right)=0.845$ (lower red horizontal line) and its approximation $\chi_{p}=0.85$ as determined from Eq. (3.4) (upper green horizontal line). 
We now use $S_{p}$ to approximate all four in-plane spin parameters, and we are free to distribute the precession spin appropriately between the two black holes in the binary. Motivated by the fact that the in-plane spin of the smaller black hole becomes more and more negligible with increasing mass ratio, we assign the precession spin completely to the larger black hole, and define the dimensionless precession spin parameter as

$$
\chi_{p}:=\frac{S_{p}}{A_{2} m_{2}^{2}} .
$$

For a small subset of configurations $\chi_{p}$ does not respect the Kerr limit of $\chi_{i} \leq 1$, i.e., when $S_{2 \|}$ and $S_{1 \perp}$ are both large. However, we find for the random sample of configurations studied in Sec. V that this is rare: we find $\sim 3 \%$ of such configurations for $q=1$ binaries, and none in our sample for $q=3$ and $q=10$.

Having chosen $\chi_{p}$ to be the approximate mean of the leading-order term in the $\mathrm{PN}$ precession equation, we expect (by construction) to see a similar evolution of the orbital plane in a system where $\chi_{p}$ is used instead of $S_{1 \perp}$ and $S_{2 \perp}$. Note that our definition of $\chi_{p}$ does not reproduce the same initial value of the precession cone opening angle, $l$; that would require that we instead focus on the average of $S_{1 \perp}+S_{2 \perp}$, and not the weighted sum in Eq. (3.3). However, we find the effect on $l$ to be small, and we also expect that it is less important to correctly model $l$ than the precession angle $\alpha$ due it its effect on the phase. This is illustrated for one generic case in Fig. 6. We see that precession angles obtained from a configuration, where the in-plane spins are replaced by $\chi_{p}$ on the larger black hole, indeed represent the average precession of the full generic system.

There are two situations where we expect that applying a spin of $\chi_{p}$ to the larger black hole may not adequately capture the average precessional motion of the corresponding full system.

One is when the precession is dominated by the in-plane spin of the smaller black hole. In these cases $\chi_{p}$ again reproduces the correct contribution to the precession equation (3.2), but the initial value of $\imath$ may differ more substantially from the correct value. In particular, $l$ is typically small now, and the oscillations in the parallel and perpendicular spin components (shown in Figs. 3 and 4) are now comparable to their mean values. An example is shown in Fig. 7. We will see in Sec. V that the waveforms nonetheless agree well in most cases, and for a wide range of binary orientations and GW polarizations.

By solving $S_{p}-A_{1} S_{1 \perp}=0$ for each mass ratio one can define the minimal in-plane spin on the larger black hole as a function of $\chi_{1 \perp}$ (the in-plane component of the smaller black hole) such that the precession is dominated by $\chi_{1 \perp}$. For mass ratio $q=3$ and a maximal in-plane spin of $\chi_{1 \perp}=1$, any in-plane spin $\chi_{2 \perp} \leq 0.289$ yields a system that is precession dominated by the smaller black hole; for $q=10$ this value drops to $\chi_{2 \perp} \leq 0.079$, showing that the
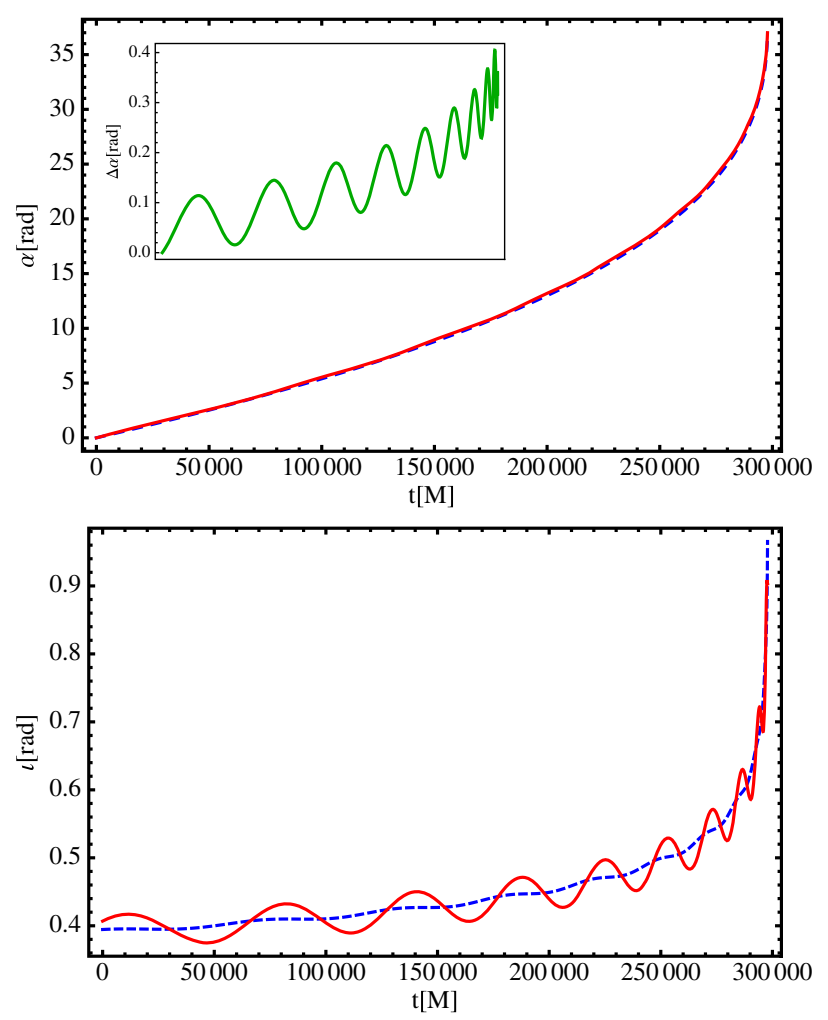

FIG. 6 (color online). The top panel shows $\alpha(t)$ for the generic configuration $\left\{q=3, \vec{\chi}_{1}=(0.4,-0.2,0.3), \vec{\chi}_{2}=(0.75,0.4,-0.1)\right\}$ (red) and the corresponding configuration utilizing $\chi_{p}$ given by $\left\{q=3, \vec{\chi}_{1}=(0 ., 0 ., 0.3), \vec{\chi}_{2}=(0.85,0 .,-0.1)\right\}$ (blue, dashed). Since the two curves are not distinguishable over that time scale, the inset shows the difference $\Delta \alpha$ as a function of time. The bottom panel compares the evolution of the opening angle of the precession cone $l(t)$. Both graphs reveal that the approximation discards the spin-spin couplings in the plane and therefore nutation effects (the visible oscillations).

fraction of binaries that are precession dominated by the smaller black hole decreases with increasing mass ratio.

The second group of configurations where $\chi_{p}$ will not adequately approximate the precession dynamics are those where there is little or no relative rotation of the in-plane spins in the orbital plane. This occurs when both constituent masses are (almost) equal, i.e., $q \simeq 1$. Then the spins remain approximately locked and the averaging that motivates $\chi_{p}$ no longer applies. The appropriate choice of inplane spin magnitude in these cases would be the sum of the two in-plane spin vectors, which remains roughly constant [25], and so $\chi_{p}$ tends to underestimate the in-plane spin contribution. The precession term for varying mass ratio is illustrated in Fig. 8. We see that, as expected, for the equal-mass case $\chi_{p}$ underestimates the average precession of the system. We see, however, that already at mass ratio $q=1.2, \chi_{p}$ is a good estimator of the precession even for mass ratios close to equal mass.

So far, we have explored the phenomenology of a single spin parameter $\chi_{p}$ to estimate the average precession in a 

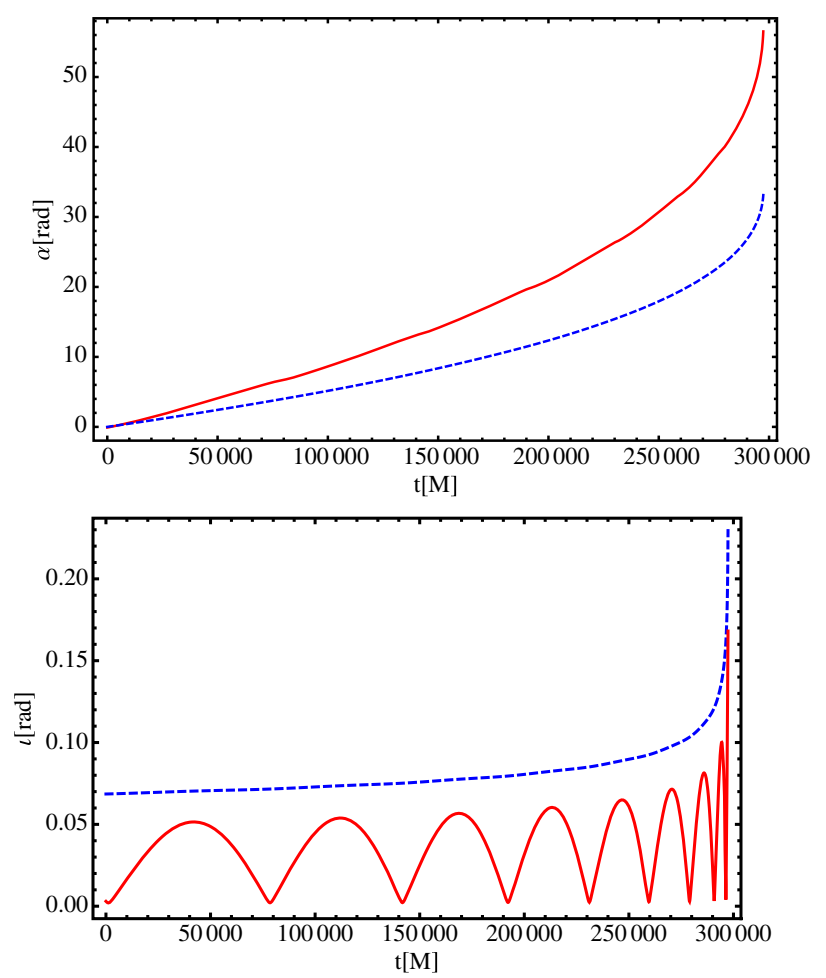

FIG. 7 (color online). The top panel shows $\alpha(t)$ for the case $\left\{q=3, \vec{\chi}_{1}=(0.38,0.319,-0.079), \vec{\chi}_{2}=(-0.036,-0.036\right.$, $-0.012)\}$ (top solid, red curve) and the corresponding configuration using $\chi_{p}$ given by $\left\{q=3, \vec{\chi}_{1}=(0 ., 0 .,-0.079)\right.$, $\left.\vec{\chi}_{2}=(0.143,0 .,-0.012)\right\}$ (bottom dashed, blue curve); the bottom panel compares the evolution of the opening angle of the precession cone $t(t)$. Both graphs highlight that in this case $\chi_{p}$ does not capture the precession of the system correctly.

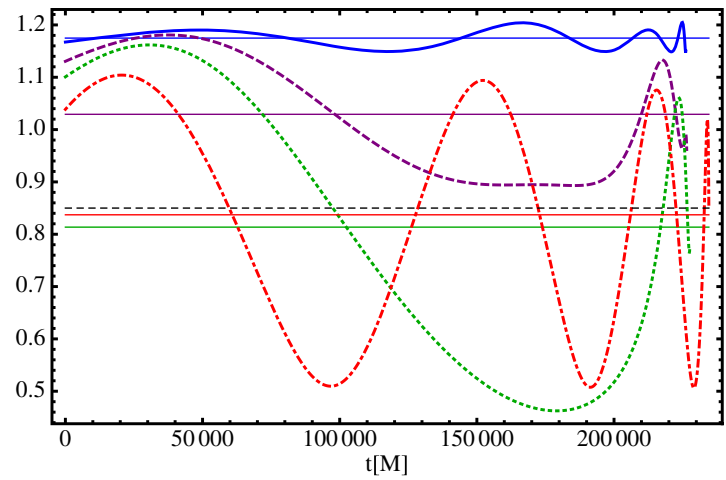

FIG. 8 (color online). The panel shows the precession term $\left\|A_{1} \vec{S}_{1 \perp}+A_{2} \vec{S}_{2 \perp}\right\| /\left(A_{2} m_{2}^{2}\right)$ and its mean as a function of time for four different mass ratios $q$ : equal-mass (blue, solid), $q=1.1$ (purple, dashed), $q=1.2$ (green, dotted) and $q=1.5$ (red, dot-dashed). The mean value for $q=1$ is 1.175 . For this spin configuration, however, Eq. (3.4) yields $\chi_{p}=0.85$ (indicated by the dashed horizontal line). We see that at a small mass ratio of $q=1.2, \chi_{p}$ is already a good estimator of the average precession. generic system and saw good agreement when considering precession-related geometric quantities like the precession angles. However, keeping our goal of modelling precessing waveforms with a smaller set of physical parameters in mind, we need to investigate and quantify the agreement between fully generic waveforms and their parameterreduced counterparts. This will be the goal of the subsequent sections.

\section{ACCURACY OF THE PRECESSION SPIN APPROXIMATION: METHODOLOGY}

We now assess the quality of our precession parametrization for PN inspiral waveforms. Our approach is to compare a large number of generic inspiral waveforms at three mass ratios, $q=1,3,10$, to a family of corresponding reduced-parameter waveforms where the initial in-plane spin components are replaced by $\chi_{p}$ applied to the larger black hole. We are interested only in the effectiveness of $\chi_{p}$ to approximate the precession, and so we use the same values for the masses and initial values of the parallel spin components $\chi_{i \|}$.

\section{A. Reduced-parameter waveforms}

We compare a given binary configuration with a full set of physical parameters with a corresponding configuration with a reduced set of physical parameters, defined by the mapping of the dimensionless spins as follows:

$$
\begin{aligned}
\left(\chi_{1 x}, \chi_{1 y}, \chi_{1 z}\right) & \mapsto\left(0,0, \chi_{1 z}\right), \\
\left(\chi_{2 x}, \chi_{2 y}, \chi_{2 z}\right) & \mapsto\left(\chi_{p}, 0, \chi_{2 z}\right),
\end{aligned}
$$

where we have defined the spins with respect to $\hat{L} \equiv \hat{z}$ in a Cartesian coordinate system. Hence, the reduced model parameters are $q, \chi_{1 \|}, \chi_{2 \|}$ and $\chi_{\mathrm{p}}$.

This does not define a bijective map: various combinations of different physical spins $\vec{S}_{1}, \vec{S}_{2}$ can yield the same set of $\left\{\chi_{1 \|}, \chi_{2 \|}, \chi_{\mathrm{p}}\right\}$ despite being physically completely different configurations. Therefore, all configurations for one set of model parameters $\left\{q, \chi_{1 \|}, \chi_{2 \|}, \chi_{p}\right\}$ do not define a single configuration but an approximate equivalence class of precessing systems, i.e., various generic configurations map to the same point in the manifold of reducedparameter configurations. If we are correct in assuming that all of these configurations agree well with each other, then this has implications for GW observations: we will be able to more easily measure the combination $\chi_{p}$ than the individual in-plane black-hole spins.

In order to assess whether this approximation indeed holds, we compute waveforms by integrating the set of $\mathrm{PN}$ equations given in Appendix A. We then compute matches between waveform strains of the generic configuration and its corresponding reduced-parameter configuration for various binary inclinations $\theta$ and GW polarization 
angles $\psi$. Henceforth, motivated by the terminology of GW searches, we will refer to the full-parameter configuration as signal and to the reduced-parameter one as model.

\section{B. Generic match}

The agreement between two waveforms is commonly quantified by the noise-weighted inner product between the two signals [44]. In the case of the real-valued detector response, $h_{\text {resp }}^{S}(t), h_{\text {resp }}^{M}(t) \in \mathbb{R}$ (where the superscripts distinguish signal and model), the match is commonly defined as

$$
\begin{aligned}
\left\langle h_{\text {resp }}^{S} \mid h_{\text {resp }}^{M}\right\rangle & =2 \int_{-\infty}^{\infty} \frac{\tilde{h}_{\text {resp }}^{S}(f) \tilde{h}_{\text {resp }}^{M *}(f)}{S_{n}(|f|)} d f \\
& =4 \operatorname{Re} \int_{0}^{\infty} \frac{\tilde{h}_{\text {resp }}^{S}(f) \tilde{h}_{\text {resp }}^{M *}(f)}{S_{n}(|f|)} d f .
\end{aligned}
$$

Here, $S_{n}$ is the noise spectral density of the detector, $\tilde{x}$ denotes the Fourier transform of $x$, and $x^{*}$ is the complex conjugate of $x$.

Note that the conversion from Eq. (4.2) to Eq. (4.3) relies on $\tilde{h}_{\text {resp }}(-f)=\tilde{h}_{\text {resp }}^{*}(f)$ which is always true for realvalued signals. Here we find it more convenient, however, to work directly with a commonly used complex waveform strain that combines both waveform polarizations,

$$
h=h_{+}-i h_{\times} .
$$

With the introduction of a polarization angle $\psi$, we can relate both waveform representations to each other via

$$
\begin{aligned}
h_{\text {resp }}(t) & =\cos (2 \psi) h_{+}(t)+\sin (2 \psi) h_{\times}(t) \\
& =\operatorname{Re}\left[h(t) e^{i 2 \psi}\right] .
\end{aligned}
$$

Note that our definitions of $h_{+}$and $h_{\times}$differ slightly from similar expressions in the literature [see, e.g., Eq. (55) in Ref. [45]] in the respect that we leave an overall factor that depends on the orientation between detector and source as part of the definitions of $h_{+}$and $h_{\times}$, while $\psi$ explicitly governs a relative rotation in the detector plane.

Our goal is to calculate the inner product between signal and model and optimize it over the model polarization angle and a relative time shift in an efficient way. We find a convenient formulation of the inner product in terms of the complex strains by inserting Eq. (4.6) into Eq. (4.2), which finally yields

$$
\begin{aligned}
\left\langle h_{\text {resp }}^{S} \mid h_{\text {resp }}^{M}\right\rangle= & \operatorname{Re} \int_{-\infty}^{\infty} \frac{\tilde{h}^{S}(f) \tilde{h}^{M *}(f)}{S_{n}(|f|)} e^{2 i\left(\psi_{S}-\psi_{M}\right)} d f \\
& +\operatorname{Re} \int_{-\infty}^{\infty} \frac{\tilde{h}^{S}(f) \tilde{h}^{M}(-f)}{S_{n}(|f|)} e^{2 i\left(\psi_{S}+\psi_{M}\right)} d f .
\end{aligned}
$$

The details of the derivation are given in Appendix B, where we also provide explicit expressions to optimize over $\psi_{M}$ (for a given signal polarization $\psi_{S}$ ) analytically.

Note that nonprecessing signals under the adiabatic assumption have all information contained on one side of the frequency spectrum, and hence the second term in Eq. (4.7) vanishes. Here, however, we do not make this assumption about the (precessing) signals; in fact, for orientations where the GW strain is not dominated by only one mode and precession features become important, we have to take into account both contributions in Eq. (4.7) to obtain the correct inner product.

The results presented in the next section are all formulated in terms of the match $\mathcal{M}$, which we define as the inner product (4.7) normalized by both signal powers and optimized over a relative time shift, the polarization angle $\psi_{M}$ of the model, and the azimuthal angle $\varphi_{M}$ in the spinweighted spherical harmonics of the model [see Eq. (4.8) for more details]. For details of the algorithm, we refer once again to Appendix B. Alternative approaches to similar problems have been introduced before in Ref. [7] and were extended in Refs. [46,47], but these relied on the construction of an orthogonal basis and expressed the results in terms of matches that were maximized or minimized over $\psi_{S}$. Here, however, we prefer to directly use the information from the complex GW strains across the entire frequency spectrum as this is what we obtain from the PN integration.

Matches (very) close to unity indicate an accurate approximation of the full signal, while any deviation from unity quantifies the degree of disagreement between model and signal. There are various application-dependent thresholds one could consider for $\mathcal{M}$, some being based on the distinguishability between model and signal, others translating mismatches to a loss in sensitive volume [48]. For simplicity, we will use $\mathcal{M}=0.965$ as a reference value, as this number is frequently used in the GW literature to mark the $10 \%$ loss in sensitive volume. We remark, however, that we are not explicitly addressing the question of detecting the signal with our proposed model. We deliberately refrain from optimizing the match over all intrinsic source parameters (which would be a meaningful strategy to quantify the detection efficiency); instead, we quantify the agreement for fixed source parameters (with the exceptions pointed out above) because we are predominantly interested in whether our reduced-parameter model introduced in Secs. III and IV A faithfully represents the full-parameter signals.

In the following, we quantify the agreement between the $(\ell=2)$-waveform strain of the signal

$$
h(t ; \theta, \varphi)=\sum_{m=-2}^{2} h_{2 m}(t) Y_{2 m}^{-2}(\theta, \varphi),
$$

by exploring the match $\mathcal{M}$ against the model as a function of the binary inclination $\theta$ and the signal polarization $\psi_{S}$ 
for a total binary mass of $M=12 M_{\odot}$ with a GW starting frequency of $20 \mathrm{~Hz}$ and a cutoff frequency of $366 \mathrm{~Hz}$. We use the early aLIGO noise curve [49].

\section{ACCURACY OF SIMPLIFIED PRECESSING INSPIRAL WAVEFORMS: RESULTS}

In the following we perform two classes of tests of our reduced-parameter model. We first test the $\chi_{p}$ parametrization on a selected set of configurations where one or both black holes have extremal spins: we vary the relative orientation of the in-plane spins of the signal configuration (Sec. VA 1), the magnitude of one of the in-plane components (Sec. VA 2), and assess the influence of the parallel spin components (Sec. VA 3). Having tested the parametrization in what we consider to be extreme cases, we then analyze in Sec. V B a large sample of configurations with three different mass ratios, $q=1,3,10$, with randomly chosen spin magnitudes and orientations, and a selection of binary orientations and polarizations.

We emphasize that the faithfulness we calculate is the lower bound for the model's detection effectualness as no optimizations over physical parameters are performed; if we were to optimize over physical parameters as done in a GW search, the resulting fitting factor would by definition be larger (or the same). The results show very strong evidence in favor of the reduced parametrization to capture the dominant precession effects.

\section{A. Selected test cases}

To test the effectiveness of the reduced parametrization, we first explore double-spin binaries with either one or two maximally spinning black holes. In the following, we

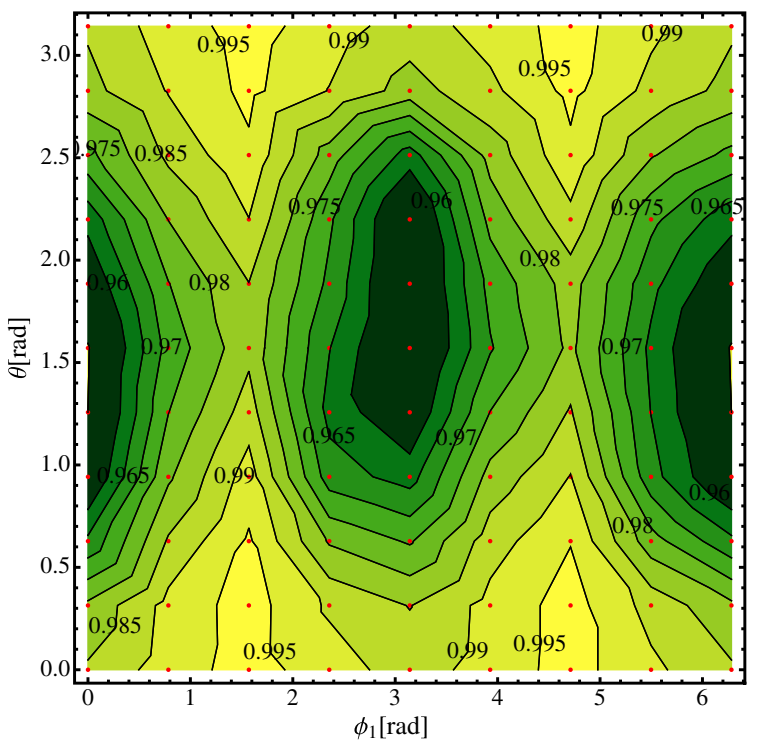

analyze various properties of these particular configurations for the mass ratio $q=3$.

\section{Relative in-plane spin orientation}

The first investigation concerns the influence of the relative orientation of the spins in the plane. Apart from the spin-spin terms in the PN evolution equations, the relative orientation of the spins has no impact on the waveform at quadrupole order. In that sense, we are now testing the influence of the spin-spin terms.

We first fix $\vec{\chi}_{2} \equiv(1,0,0)$ and vary the orientation of $\vec{\chi}_{1}=\left(\cos \phi_{1}, \sin \phi_{1}, 0\right)$ with $\phi_{1} \in[0,2 \pi]$ and $\Delta \phi_{1}=45^{\circ}$. We then interchange the roles of $\vec{\chi}_{1}$ and $\vec{\chi}_{2}$ and vary $\phi_{2}$ in the same interval. To quantify the agreement between each rotated generic waveform and our model waveform that remains unaffected by these rotations, we compute the match between these two, respectively. We choose a set of different binary orientations $\theta \in[0, \pi]$ with $\Delta \theta=\pi / 10$, but keep the signal polarization fixed for a polarization angle $\psi_{S}=0$ and set the azimuthal orientation of the signal to $\varphi_{S}=0$. We optimize the match over the template polarization, a time shift and the angle $\varphi_{M}$ in the spinweighted spherical harmonics of the template strain.

The results are illustrated in Fig. 9. In both cases we obtain very high matches but observe 1) a mild dependence on the relative orientation in the plane and 2) a strong dependence on the binary's orientation $\theta$. The minimal match is $\mathcal{M}_{\min }=0.95$ in both cases. We find that the lowest matches are clustered around "edge-on" orientations of $\theta=\pi / 2$.

The pattern of low matches in Fig. 9 can be explained by considering the PN evolution equation (A5). The spin-spin

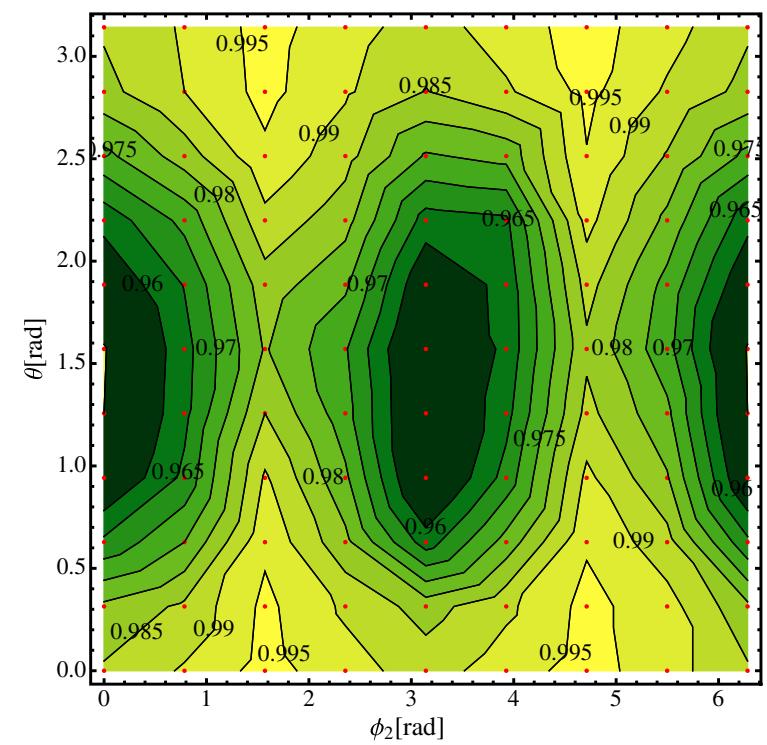

FIG. 9 (color online). The left panel shows the match contours for the extremal case with $\vec{\chi}_{2}=(1,0,0)$ and varying in-plane orientation of $\vec{\chi}_{1}$, while the right panel shows the contour for $\vec{\chi}_{1}=(1,0,0)$ and varying orientation of $\vec{\chi}_{2}$ as a function of the binary orientation $\theta$. The red dots mark the actual points at which the matches are evaluated. 
$\left(\vec{S}_{1} \cdot \vec{S}_{2}\right)$ contribution vanishes completely in the reducedparameter system which in this case only has one nonvanishing spin. However, the full system does have a spin-spin contribution, and this is maximized at the beginning of the evolution when $\phi=n \pi$. In these cases the inspiral rate, and therefore the GW phase evolution, will differ during the early part of the evolution. The evolution of all orbital components is slower at earlier times, and so the level of agreement in the early phase of the evolution has the strongest influence on the overall agreement of the two final waveforms. This explains why the matches are lowest around $\phi=n \pi$. We emphasize, however, that this is purely based on the fact that we indicate the phase when the signal enters the detector band. If we were to show spin angles at different times or frequencies, the pattern in Fig. 9 would shift. The location of the poor-match regions with respect to an arbitrary $\phi$ has no physical significance.

\section{Varying the in-plane spin magnitude}

In this section we investigate the influence of the inplane spin magnitude. We fix the relative spin orientation to $\phi_{1}-\phi_{2}=0$ in this study as we have seen earlier that initially parallel in-plane spins yield the lowest matches for certain orientations. As before, the signal polarization is fixed such that $\psi_{S}=0$ and we choose $\varphi_{S}=0$; we compute the match for various binary orientations. First, we let $\vec{\chi}_{2}=$ $(1,0,0)$ and vary the magnitude of the spin on the smaller black hole such that $\vec{\chi}_{1}=\left(\chi_{1 x}, 0,0\right)$. We then exchange the role of the two black holes and vary $\vec{\chi}_{2}=\left(\chi_{2 x}, 0,0\right)$ and set $\chi_{1 x}=1$. The contours for the matches as a function of the in-plane spin magnitude of one of the holes and the binary inclination $\theta$ are shown in Fig. 10.

We find that the magnitude of the in-plane spin of the smaller black hole is negligible up to $\left|\chi_{1 x}\right| \simeq 0.8$, and for $\left|\chi_{2 x}\right| \simeq 0.7$. The lowest matches are recovered for maximal in-plane spins on both black holes, which is consistent with the results regarding the relative orientation. Again, we can attribute decreasing matches to the growing influence of the spin-spin coupling term that is proportional to the individual spin magnitudes; our simplified model discards parts of these terms completely. We also observe additional structures in the match contours when $\vec{\chi}_{1}$ is fixed and the in-plane spin magnitude of $\vec{\chi}_{2}$ is varied, in particular for $\left|\chi_{2 x}\right| \simeq 0$.

\section{The influence of parallel spins}

In the cases we have considered so far, we have set the parallel components of the spins initially to zero so that they exhibit only small oscillations around zero throughout the inspiral. As described earlier, the precessional dynamics decouples approximately from the inspiral dynamics, and therefore in these cases we have studied precession effects with minimal spin influence on the inspiral.

We now introduce nonzero parallel spin components and therefore study our reduced parametrization for different inspiral rates. We consider the following configuration: the spin on the larger black hole is fixed and set to $\vec{\chi}_{2}=$ $(0.8,0,-0.6)\left(\chi_{2}=1\right)$; we now vary the spin of the smaller black hole $\vec{\chi}_{1}=\left(\chi_{1 x}, 0, \chi_{1 z}\right)$. The mass ratio is again $q=3$.

The results for three binary inclinations $\theta=0^{\circ}, 36^{\circ}, 90^{\circ}$ and signal polarization $\psi_{S}=0$ are shown in Fig. 11. The lowest match we obtain is $\mathcal{M}_{\text {min }}=0.826$ for the configuration with $\vec{\chi}_{1}=(-1,0,0)$. Following Eq. (4.1), the parallel components of the model waveform are the same as in the generic signal. Keeping this in mind, Fig. 11 can be interpreted as follows: if $\chi_{1 \perp}=0$, then the reduced system exactly corresponds to the generic system and we therefore obtain matches $\mathcal{M}=1$. For $\chi_{1 z}=0$ we see a decreasing
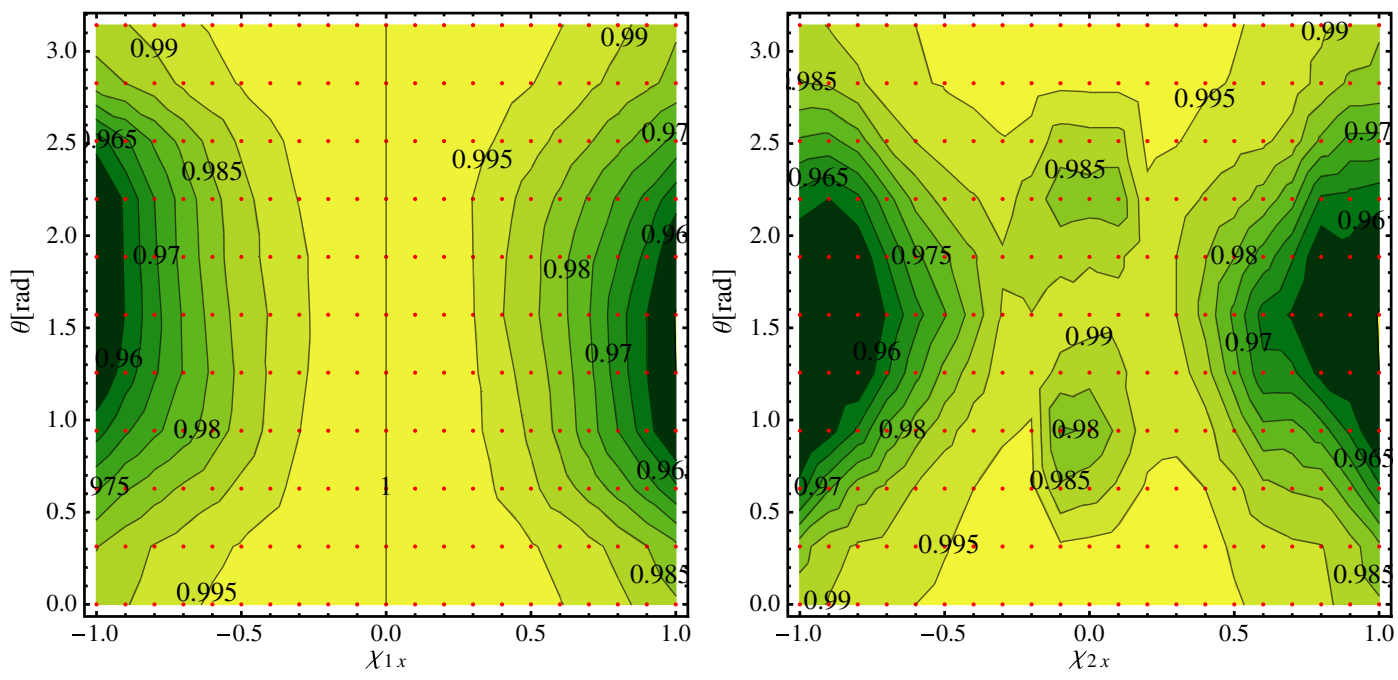

FIG. 10 (color online). The left panel shows the match for $\vec{\chi}_{1}=\left(\chi_{1 x}, 0,0\right)$ and $\vec{\chi}_{2}=(1,0,0)$ against the appropriate reducedparameter waveforms as a function of the binary orientation; the right panel shows the match for $\vec{\chi}_{1}=(1,0,0)$ and $\vec{\chi}_{2}=\left(\chi_{2 x}, 0,0\right)$ against the appropriate reduced-parameter template waveforms. The red dots mark the actual configurations used to obtain the contours. 

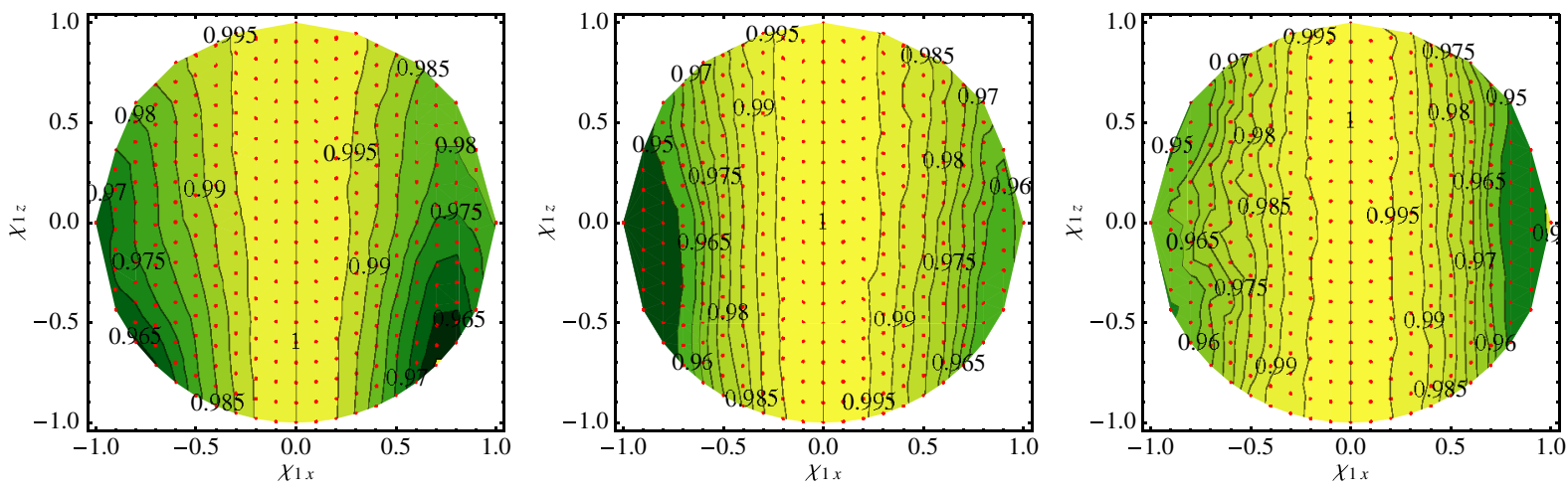

FIG. 11 (color online). The panel shows the match contours for three different binary inclinations $\left(0^{\circ}, 36^{\circ}\right.$ and $\left.90^{\circ}\right)$ for the configurations where $\vec{\chi}_{2}=(0.8,0,-0.6)$ and $\vec{\chi}_{1}=\left(\chi_{1 x}, 0, \chi_{1 z}\right)$. Each red dot represents one particular choice of $\left(\vec{\chi}_{1}, \vec{\chi}_{2}\right)$. We find that the matches drop with increasing value of $\left|\chi_{1 x}\right|$ and decrease overall with increasing inclination $\theta$.

agreement with increasing $\left|\chi_{1 \perp}\right|$ due to the neglect of the in-plane contribution to the spin-spin coupling. In between these extremes we see nearly vertical contours indicating that the mismatch is indeed dominated by the neglect of $\left(\vec{S}_{1 \perp} \cdot \vec{S}_{2 \perp}\right)$ and rather independent of the parallel spin components as these are preserved in the particular mapping and PN treatment we use.

\section{B. Statistical analysis: a random sample of precessing configurations}

Previously, we have analyzed a handful of test cases, which allowed us to extract trends along several directions in the configuration space. Further, we were able to quantify the influence of the in-plane spin-spin coupling, which is neglected in our approximation. In order to assess the goodness of the reduced-parameter model across the precessing binary parameter space, a significant sample of all possible configurations needs to be analyzed at various mass ratios. We therefore construct 10000 random binary spin configurations with uniform sampling in the dimensionless spin magnitudes $\chi_{1,2} \in[0,1]$ and the spin azimuth angles $\phi_{1,2} \in[0,2 \pi]$, for mass ratios $q=1,3$ and 10 .

We analyze the sample by quantifying the agreement between the $(\ell=2)$-waveform strain as given in Eq. (4.8) for each configuration in the sample with its corresponding reduced-parameter model $h^{M}$ by computing the match $\mathcal{M}$. As before, we optimize only over the following subset of extrinsic parameters: the polarization $\psi_{M}$ of the model waveform, the azimuth $\varphi_{M}$ in the reduced-parameter GW strain, as well as a time shift $\Delta t$; we do not optimize over the physical parameters $m_{1}, m_{2}, \vec{S}_{1}$ or $\vec{S}_{2}$. We repeat this match computation for each configuration for the signal polarization angles $\psi_{S} \in\{0 ., \pi / 8, \pi / 4,3 \pi / 8\}$ as well as for the binary orientations $\theta \in\{0, \pi / 10, \pi / 4,2 \pi / 5$, $\pi / 2,3 \pi / 5,4 \pi / 5,11 \pi / 12\}$ with $\varphi_{S}=0$. This yields 32 individual matches per configuration and a total of 320000 matches. We repeat this calculation for various mass ratios but fix the following parameters in the analysis.
We set the initial separation $r_{i}=40 M$, to obtain sufficiently long inspiral waveforms in the time domain, which are sampled at intervals of $\Delta t=10 \mathrm{M}$. We set the total mass to $M=12 M_{\odot}$. This is an ad hoc choice, but was made to allow a wide frequency range in the detector's sensitivity band, to minimize the effects of merger and ringdown and for reasons of computational cost efficiency. We fix the upper cutoff frequency to be $\mathrm{Mf}_{\text {ISCO }}=\left(\pi 6^{3 / 2}\right)^{-1}$ and use the anticipated early PSD noise curve for aLIGO [49].

\section{General results}

We present the results of our large-scale study in Fig. 12, where we show the cumulative distribution functions (CDFs) for our statistical samples: for each value of the match, the figure indicates the fraction of signals that have a match less than that value. In the left panel, this fraction is simply based on the number of matches we have calculated. In the right panel, however, we estimate the fraction of detectable signals by reinterpolating our results over a uniform grid in $\cos \theta$ and by assigning a signal-to-noiseratio-dependent volume to each signal. By comparison we see that most signals which are not well captured by our model are unlikely to be detected (e.g., edge-on signals with pronounced precession effects have a considerably smaller amplitude than less modulated face-on signals at the same distance); therefore, the right panel of Fig. 12 shows generally better results than the left panel. In order to be conservative and emphasize the modelling (i.e., amplitude-independent) focus of this paper, we shall only quote numbers obtained from the left panel of Fig. 12 below.

For mass ratios $q=1,3$ we find that less than $2 \%$ of all matches are below 0.965 , respectively, showing that the precession in the system is faithfully represented by the effective precession parameter $\chi_{p}$ for most binary configurations and orientations. For both mass ratios, more than $88 \%$ of all matches are above 0.99 . We find a difference in the CDF tails towards low matches, where the $q=1$ curve is considerably flatter than for $q=3$, which is rather 

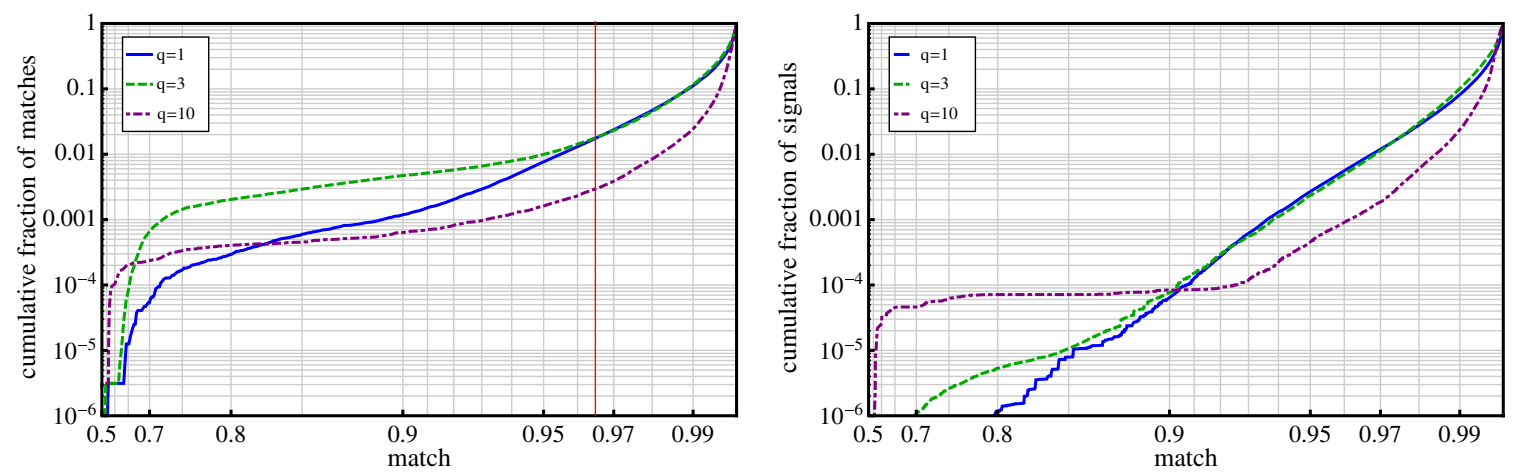

FIG. 12 (color online). The left panel shows the cumulative distribution function for all matches for the mass ratios $q=1$ (blue, solid), $q=3$ (green, dashed) and $q=10$ (purple, dot-dashed). The red vertical line indicates a match of $\mathcal{M}=0.965$. In the right panel the distribution is weighted according to the signal strength to represent the fraction of actually detectable signals.

surprising at first glance. It can be explained by the error introduced for unequal-mass cases with very little precession, which are not well captured by $\chi_{p}$. For completeness, we remark that the minimum match for $q=1$ is found at $\mathcal{M}_{\min }=0.558$ for the following spin configuration: $\vec{\chi}_{1}=$ $(0.14,0.13,0.75)$ and $\vec{\chi}_{2}=(0.12,0.22,-0.42)$. For $q=3$ we find the minimum at $\mathcal{M}_{\text {min }}=0.532$ for the configuration $\vec{\chi}_{1}=(0.53,-0.04,-0.63)$ and $\vec{\chi}_{2}=(-0.16,0.18,0.76)$.

Additionally, we have computed the matches for mass ratio $q=3$ with a random choice of $\varphi_{S}$ and obtain a $\mathrm{CDF}$ that shows no significant deviation from the result when $\varphi_{S}=0$.

As we have mentioned at the end of Sec. III, one might not have expected our $\chi_{p}$ parametrization to work accurately in the equal-mass case as the two spins are locked and therefore the binary follows the evolution of a single spin binary with a total spin magnitude $S=\left\|\vec{S}_{1}+\vec{S}_{2}\right\|=$ const. The appropriate parameter reduction for $q=1$ configurations might therefore be to put the total spin, $\vec{S}=\vec{S}_{1}+\vec{S}_{2}$ onto the larger black hole, which would be equivalent to the reduction used in the physical template family [37]. However, we find that this choice has little effect on the results in Fig. 12. This indicates that in these configurations, the impact of neglecting the spin-spin terms (by placing all of the spin on one black hole) is comparable to that of making our $\chi_{p}$ parameter reduction. As the mass ratio increases, the $\chi_{p}$ parametrization becomes more accurate, and the influence of the spinspin terms on the phase evolution decreases.

The other class of possibly problematic cases that we identified in Sec. III are those where precession is dominated by the small black hole. We find a total of 1699 of these configurations (17\%) for mass ratio $q=3$. However, only $4.7 \%$ among these matches are below the threshold $\mathcal{M}=0.965$. Further, we find that these subthreshold matches are predominantly clustered around values for $\chi_{2 \perp} \leq 0.08$. We conclude that $\chi_{p}$ faithfully represents binaries that are precession-dominated by the smaller black hole; only systems with very little precession, which make up less than $1 \%$ of our set of test cases, are not faithfully approximated for certain binary inclinations and signal polarization angles.

We expect the mapping onto the reduced-parameter waveforms to be yet even more faithful for higher mass ratios such as $q=10$. On the other hand, we now expect transitional precession to occur more often within the sensitivity band of aLIGO. In order to identify the occurrence of transitional precession, we follow Ref. [25] and define an initial angle $\measuredangle(\hat{L}, \hat{S}) \geq 164^{\circ}$ to indicate transitional precession. We find that $1.8 \%$ of the sample configurations undergo the transitional phase either completely or partially in band.

We again illustrate the results through the CDF of matches in the purple dot-dashed curve in Fig. 12. As expected, the tail is much flatter than for the low-mass-ratio end with a fraction of only $0.3 \%$ of all matches below threshold. More than $97 \%$ of cases show a match of 0.99 or better. The minimum match obtained is $\mathcal{M}_{\min }=$ 0.484 for the configuration $\vec{\chi}_{1}=-(0.56,0.48,0.06)$ and $\vec{\chi}_{2}=\{0.01,0.02,-0.60\}$, which undergoes the full transitional phase in band. The final angle between $\hat{J}$ and $(0,0,1)$ is $146.6^{\circ}$. We illustrate the details of this particular case in the next section.

\section{Special case: transitional precession}

Our random distribution of $q=10$ configurations includes some instances of in-band transitional precession. As expected, these cases give, for certain orientations and polarizations, matches significantly below threshold, some as low as $\sim 0.4$.

Transitional precession occurs when the total spin $\vec{S}$ and the orbital angular momentum $\vec{L}$ have similar magnitude but are directed nearly opposite, such that the magnitude of the total angular momentum $J$ is small. This will only occur within the frequency band of ground-based GW detectors for a narrow range of physical parameters. For a small set of configurations, the binary starts in a simply precessing 

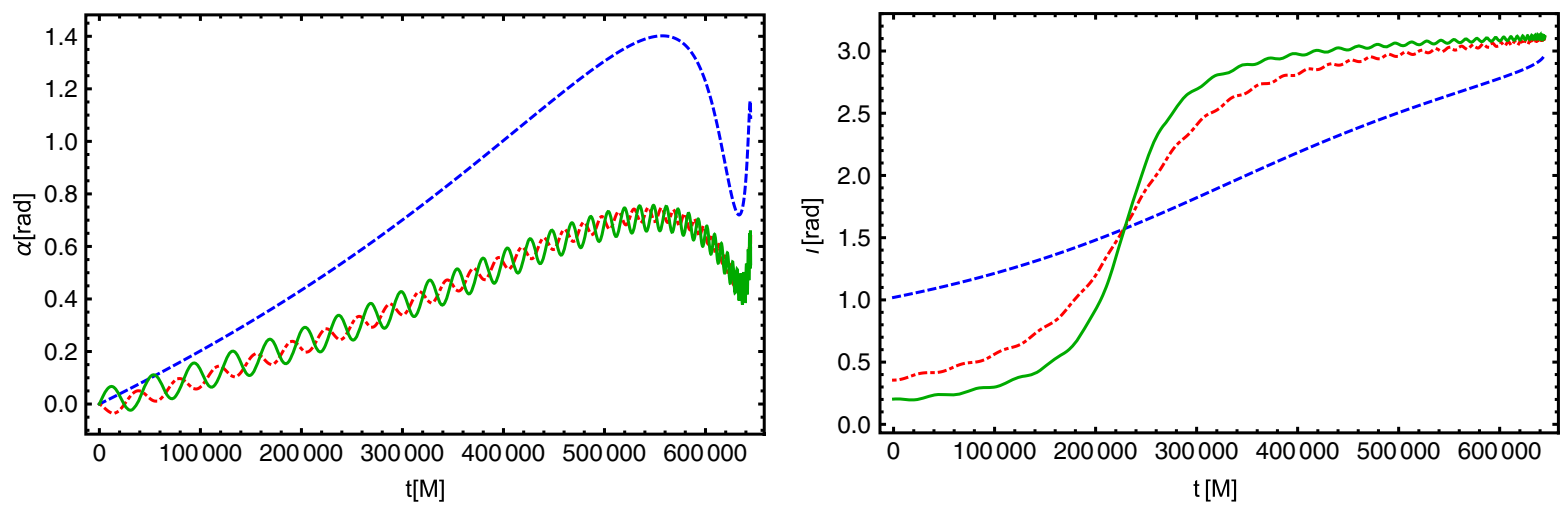

FIG. 13 (color online). The left panel shows the PN evolution of the precession angle $\alpha$ for the transitional precession case described in the text (red, dot-dashed) as well as $\alpha(t)$ for the corresponding reduced-parameter template (blue, dashed). The right panel compares the two precession cone opening angles. It is clear from those graphs that the mapping does not faithfully reproduce transitional precession. The green (solid) curves show the angles for a reduced-parameter system, where the precession is associated with the smaller black hole $m_{1}$, which appear to be closer to the angles in the generic system (red, dot-dashed).

phase, then undergoes a transitional phase, and, if it has not yet merged, returns to a state of simple precession.

Figure 13 shows in red the evolution of the precession angles $(l(t), \alpha(t))$ for the transitional configuration described previously. The true physical system has initial spins $\vec{\chi}_{1}=-(0.56,0.48,0.06)$ and $\vec{\chi}_{2}=(0.01,0.02,-0.60)$, while the corresponding reduced-parameter configuration has initial spins $\vec{\chi}_{1}=-(0,0,0.06)$ and $\vec{\chi}_{2}=$ $(0.06,0,-0.60)$. The comparison of the two precession angles $\alpha$ and $\imath$ from the transitional configuration with its corresponding model configuration reveals a strong disagreement. This can be explained as follows: for transitional precession to also occur in the reduced-parameter configuration, it is crucial that the parallel component of the total spin is close to $S_{\|}$in the generic configuration. Since we fix the parallel spin components in the mapping, the fulfillment of this condition is guaranteed. At the same time, however, $S_{\perp}$ must also be similar to the full-parameter system. If it is too large, the transitional phase occurs at later times; if it is too small, the transition is shifted to earlier times. By construction, $\chi_{p}$ corresponds to an average in-plane spin, which does not necessarily correspond to $S_{\perp}$ of the generic system. We conclude that the faithful representation of transitional precession is highly sensitive to the initial value of $S_{\perp}$, but note that a different value of $\chi_{p}$ is in principle capable of capturing transitional precession. In the green curves in Fig. 13 we illustrate this by placing the precession spin on the smaller black hole, but similar results could also be achieved by optimizing over $\chi_{p}$ in our standard construction.

\section{On the goodness of $\chi_{p}$}

The results obtained so far suggest that the single spin parameter $\chi_{p}$ faithfully represents the precession in a given generic double-spin system. What we have not yet investigated, however, is the goodness of this parameter, i.e., whether the particular definition of $\chi_{p}$ that we have chosen is ideal. We can investigate this by determining the magnitude of the large black hole's in-plane spin that yields the best agreement with the signal waveform, and compare that with our estimate of $\chi_{p}$. To do so, we determine the match of a single generic case with a series of reduced-parameter configurations, where we vary the value of $\chi_{p}$. Previously, we have seen that the match strongly depends on the inclination $\theta$ of the binary as well as the polarization angle $\psi_{S}$ of the signal. We therefore repeat the analysis for several values of $\theta$ and $\psi_{S}$. The results are illustrated in Fig. 14 for the same configuration as depicted in Fig. 6, $\left\{q=3, \vec{\chi}_{1}=(0.4,-0.2,0.3)\right.$, $\left.\vec{\chi}_{2}=(0.75,0.4,-0.1)\right\}$.

For an optimally oriented binary (i.e., $\theta=0$ ) the match depends only weakly on the explicit value of $\chi_{p}$. This is consistent with our expectation that a large fraction of optimally oriented precessing binaries is well represented by aligned-spin binaries [19,34,50-52]. For larger inclinations $\theta$, however, the match becomes more strongly dependent on $\chi_{p}$. We find that the best match is indeed obtained for a $\chi_{p}$ value close to the theoretically predicted one, indicating that $\chi_{p}$ does provide a meaningful parametrization of the precession and allows for a faithful representation of a generic system in particular for large inclinations. This needs to be investigated in more detail for a larger number of precessing configurations, and for a full optimization over all physical parameters, which we defer to future work.

Note the different scales in the upper and lower panels of Fig. 14. These suggest that our ability to measure precession effects will depend strongly on the orientation of the binary. For binaries with $\theta \approx 0$, it will be more difficult to distinguish that a binary is precessing, than for one with larger orientations. Our ability to measure not just whether the binary is precessing, but also the value of $\chi_{p}$, will of course also depend on all correlations of $\chi_{p}$ with other 

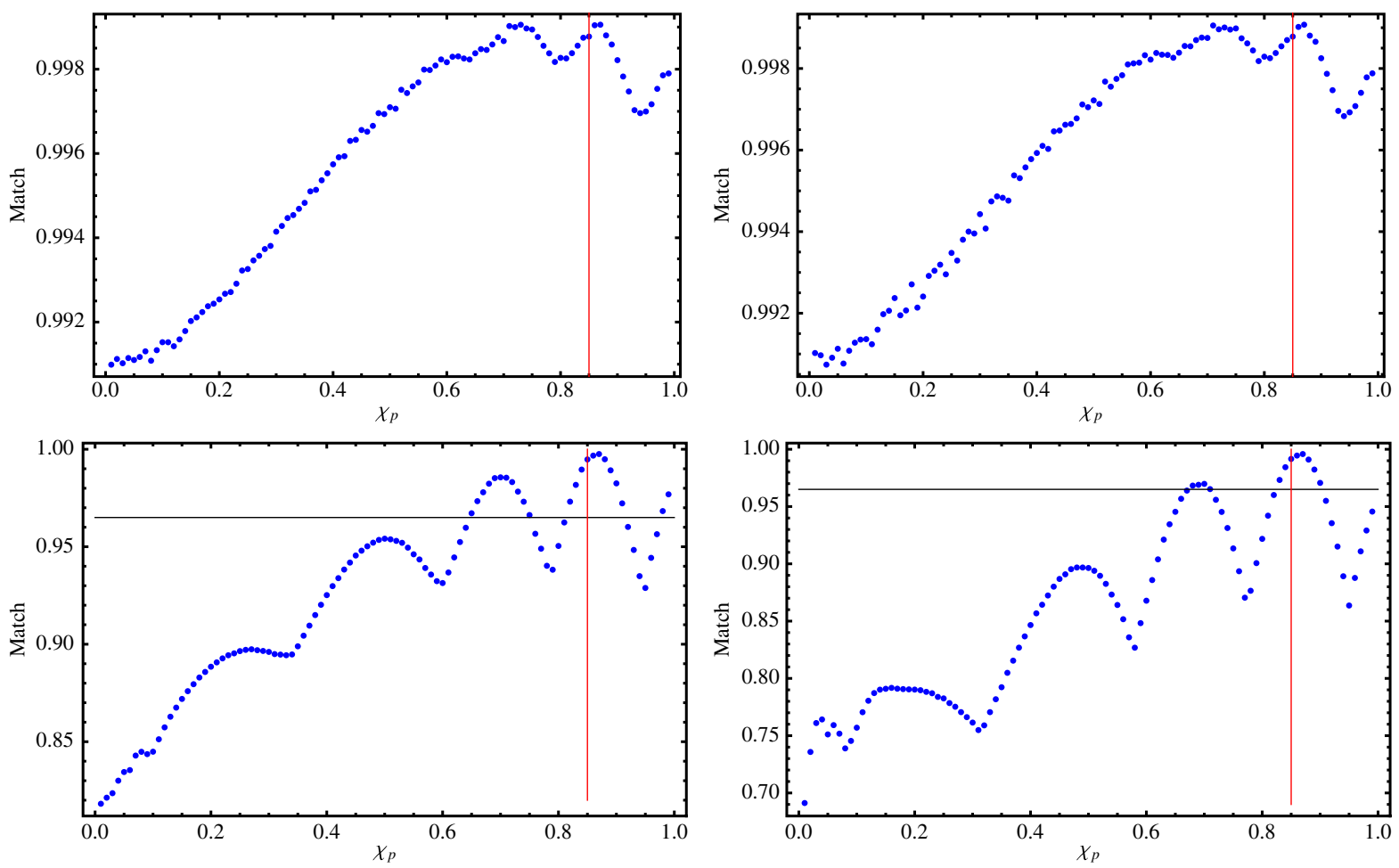

FIG. 14 (color online). The four panels show the matches for the case depicted in Fig. 6 with a series of reduced-parameter configurations with varying $\chi_{p}$ for four different pairs of binary orientation and signal polarization $\left(\theta, \psi_{S}\right)$; these are as follows from the top left to the bottom right: $(0, \pi / 8),(0, \pi / 4),(\pi / 4, \pi / 8)$ and $(\pi / 2, \pi / 8)$. The red vertical line indicates the theoretical $\chi_{p}$ value; the black horizontal line in the lower two panels indicates the threshold of $\mathcal{M}=0.965$. We find a strong dependence of the match on the value of $\chi_{p}$ for growing inclinations, where waveform modulations become more pronounced. Moreover, the theoretical $\chi_{p}$ value is very close to the value yielding the maximal match.

parameters (which we keep fixed here) and on the errors in the waveform model, but this requires a more in-depth study.

It follows from the discussion after Eq. (3.3) that, if we were able to accurately measure $\chi_{p}$, then for most configurations this would translate into an accurate measurement of the in-plane spin magnitude of the larger black hole. Conversely, the in-plane spin of the smaller black hole would be poorly constrained. This is consistent with the results given in Ref. [53], where the spin of the larger black hole is in some cases measured to within $10 \%$.

\section{COMPARISON WITH OTHER REDUCED-PARAMETER FAMILIES}

In this work we have considered one choice of parameter-reduced waveform family, i.e., we have replaced the black-hole spin components that lie in the orbital plane (at some arbitrarily chosen time), with a binary with the same physical parameters, except that now only the larger black hole has any in-plane spin, and its magnitude is $\chi_{p}$, the effective precession spin parameter we defined in Sec. III. The purpose of this study has been to determine whether the dominant precession effects can be captured by a single "precession parameter," and our results suggest that in most cases it can.

We can also infer from these results that our reducedparameter waveform family may be a good candidate for use in template banks in a search for precessing binaries. We defer a detailed study of the efficacy of the $\chi_{p}$ waveforms in searches to future work; in particular, such a study would require calculations of fully optimized matches (fitting factors). However, it is natural to ask how the $\chi_{p}$ family compares to reduced-parameter families that have been suggested in previous work, or how those parameter reductions might be combined with our $\chi_{p}$ approximation.

We consider three families: the single-spin "physical template family" [37], and two waveform families that also use the "effective spin" approximation to reduce the two spin components parallel to the orbital angular momentum to a single parameter, $\chi_{\text {eff }}$.

\section{A. Comparison with the physical template family}

Buonanno et al. [37] suggested in 2004 a single-spin precessing waveform family that is effectual in detecting generic double-spin precessing binaries. Their quasiphysical template family (PTF) exhibited very high fitting 
factors across a wide range of configurations. We do not calculate fitting factors here, and therefore cannot make a direct comparison with the PTF, but comparing our partially optimized matches with the PTF will give us an indication of how they may compare in terms of parameter estimation.

Let us first point out the differences between the two waveform families. Based on the approximate decoupling between the inspiral and precession dynamics, we suggest that the inspiral is well described by the two parallel spin components, whereas the precession can be encapsulated in a single complementary spin parameter. This yields a double-spin system with three spin parameters as given in Eq. (4.1). The PTF, on the other hand, assigns the total spin $S$ of the double-spin configuration to the larger black hole, resulting in a pure single-spin system, again with three spin parameters, obtained by the following map:

$$
\begin{gathered}
\vec{\chi}_{1} \mapsto(0,0,0), \\
\vec{\chi}_{2} \mapsto \frac{\vec{\chi}_{1} m_{1}^{2}+\vec{\chi}_{2} m_{2}^{2}}{m_{2}^{2}} .
\end{gathered}
$$

This mapping can be compared with the reducedparameter mapping we use in Eq. (4.1). We expect that our mapping will allow us to correctly capture the inspiral rate (through the two parallel spin components), while $\chi_{p}$ will drive the appropriate precession. In contrast, the PTF mapping provides only one parallel spin component, and we therefore expect that it will not capture the inspiral rate so accurately.

We now test that conjecture by calculating the match of both approximations for one comparable mass ratio $q=3$ using the same sample of generic spin configurations as in Sec. V B. We apply our proposed mapping to each configuration, as well as the PTF mapping, and compute the matches against the double-spin target signal, respectively. Figure 15 shows the cumulative distribution function for both mappings. We find that the mapping suggested by the PTF results in 53\% of all matches smaller than 0.965 , compared to only $\sim 2 \%$ for the mapping given in Eq. (4.1). We therefore conclude that the assignment of the total spin to the larger black hole does not yield a particularly faithful representation of the generic double-spin system, whereas the split into the parallel spin components $\chi_{i \|}$ and $\chi_{p}$ yields matches above threshold for $\sim 98 \%$ of all configurations.

Another way to interpret this result is that one of the three spin components in the PTF mapping is the orientation of the larger black hole's spin in the orbital plane. This orientation is approximately degenerate with the binary's orientation angle $\varphi_{S}$, and any variation in this angle has only a small effect after optimizing over the corresponding model angle $\varphi_{M}$. This leaves the PTF model with only two other spin parameters with which to capture the waveform, while our $\chi_{p}$ model has three.

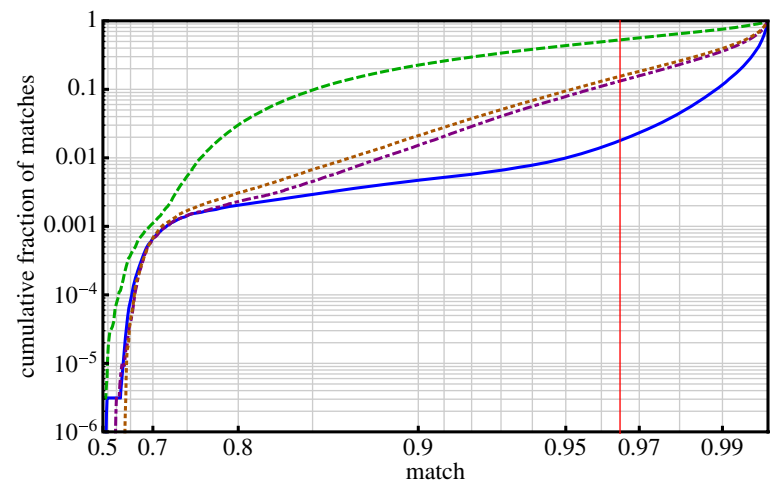

FIG. 15 (color online). Comparison of the $\chi_{p}$ model with alternative parameter reductions. Our $q=3$ results from Fig. 12 are repeated in the blue curve. Also shown are the PTF parametrization (green, dashed), the single parallel spin $\chi_{\text {eff }}$ applied to the larger black hole only (purple, dot-dashed) and $\chi_{\text {eff }}$ as parallel spin on both black holes (orange, dotted). See text for more details.

We shall investigate in the next section whether a parameter reduction from three to two spin parameters completely accounts for the loss in accuracy observable in Fig. 15 for the PTF model.

\section{B. $\chi_{\text {eff }}$ parametrization of the inspiral rate}

In the analysis presented so far, we have kept the parallel spin components in the reduced-parameter system the same as in the full-parameter system. We now investigate an additional parameter reduction, where we now replace the two parallel spin components with the effective inspiral spin, $\chi_{\text {eff }}$, as was used in several phenomenological IMR models [19,21], including a precessing-binary model [33]; its efficacy was studied in more detail in Ref. [36]. Since we use PN inspiral waveforms, we use the extended version of the effective inspiral spin as defined by [34],

$$
\chi_{\text {eff }}=\frac{1}{2}\left(1-\frac{76 \eta}{113}\right)\left(\chi_{1 \|}+\chi_{2 \|}\right)+\frac{1}{2} \frac{\delta M}{M}\left(\chi_{1 \|}-\chi_{2 \|}\right),
$$

where $\delta M=m_{1}-m_{2}$ and $\eta=m_{1} m_{2} / M^{2}$ is the symmetric mass ratio.

We consider two parameter reductions with $\chi_{\text {eff }}$. One is to place all of the spin on the larger black hole. If we once again define our configurations with respect to $\hat{L} \equiv \hat{z}$, this reduction is then given by

$$
\vec{\chi}_{1} \mapsto(0,0,0), \quad \vec{\chi}_{2} \mapsto\left(\chi_{p}, 0, \frac{113 \chi_{\mathrm{eff}}}{(113-76 \eta)}\right) .
$$

This is the choice that is made in the construction of the PhenomP precessing-binary model [33]. The second option is to choose 


$$
\chi_{i \|}=2 \chi_{\mathrm{eff}}\left(1-\frac{76 \eta}{113}-\frac{\delta M}{M}\right)^{-1}
$$

as the parallel spin component on each black hole.

We investigate the faithfulness of these two parametrizations using the same $q=3$ configurations as in Sec. V B. Figure 15 illustrates the results in the form of the cumulative fraction of matches as a function of the match. We see that both $\chi_{\text {eff }}$ models show an improved performance compared to the PTF, but a worse performance than the original three-spin-parameter model. We now find $\sim 14 \%$ and $\sim 16 \%$ of all matches below the threshold, respectively. With the reduction of the parallel spin components, both the accuracy in modelling the secular phasing is slightly decreased, and the precession dynamics is also affected, as it is governed by the total spin (in particular the initial precession cone opening angle) rather than the effective spin combination. The sum of both effects leaves us with $\sim 15 \%$ of matches below threshold.

We note that this loss in accuracy is generally a function of the mass ratio $q$. However, a simple calculation shows that for a constant choice of $\chi_{\text {eff }}$, the uncertainty in the total spin, introduced by the ambiguous distribution of the individual parallel spin components between the two black holes, scales approximately with $(q-1) /(1+q)^{2}$, which is maximal for $q=3$. Therefore we expect our results for this mass ratio to indicate the worst performance of a $\chi_{\text {eff }}$ parametrization.

\section{DISCUSSION}

In the analysis presented here, we have explored the possibility of parametrizing the precession in generic double-spin black-hole binaries with only one precession spin parameter. The inspiral and precession dynamics approximately decouple, and the precession is determined predominantly by the spin components that lie in the orbital plane. The leading-order precession effects in PN theory indicate that these in-plane spin components rotate in the orbital plane at different rates and that their magnitudes show only small variations. This motivates a spin parameter, $\chi_{p}$, which is defined as a simple mean of the in-plane spins.

We have tested the effectiveness of this parametrization by constructing a reduced-parameter family of binary waveforms, where we replaced the in-plane spin components by assigning $\chi_{p}$ as the in-plane spin of the larger black hole [see Eq. (4.1)]. We have quantified the accuracy of these reduced-parameter waveforms for the extreme case of one or two maximally spinning black holes, with respect to variations of the relative in-plane spin orientations (Sec. VA 1), the in-plane spin magnitude (Sec. VA 2), and the magnitude of the spin components parallel to the orbital angular momentum that affect the inspiral rate (Sec. VA 3). In most cases the reduced-parameter waveforms agree well with the full-parameter signals, with the worst agreement occurring when the line of sight between the source and the detector is perpendicular to the binary's total angular momentum. We have identified this disagreement to mainly originate from partially neglecting spin-spin interactions in our model.

We compared our waveform family against a random sample of 10000 configurations at mass ratios $q=1,3,10$. The agreement of our reduced-parameter model with each full-parameter configuration is shown in Fig. 12 which indicates good agreement for a large fraction of configurations. This is even true in the equal-mass case, where the in-plane spins rotate at the same rate, and therefore averaging over the spin orientation becomes invalid and $\chi_{p}$ no longer approximates the true average precession rate. However, the error in this approximation appears to be no greater than the error in neglecting spin-spin effects.

The efficacy of the precession parameter has implications for $\mathrm{GW}$ measurements. If the dominant precession effects can be captured with only one spin parameter, then this indicates that it will be difficult to distinguish the individual spin vectors in a GW observation. We already know that if the binary's total angular momentum is oriented towards the detector, then the precession will have only a minimal effect on the waveform, and so the precession will be difficult to detect. But even in binaries where the total angular momentum has a large inclination angle with respect to the detector's line of sight, and precession effects are strong, it may be difficult to identify both of the individual in-plane spin magnitudes. However, as discussed following Eq. (3.3), for many configurations, if we can accurately measure the parallel spin components and $\chi_{p}$, then we will be able to accurately measure the spin magnitude of the larger black hole.

The results of this paper add to our overall understanding of the dominant parameters that will be measurable in GW observations of binary coalescences. In aligned-spin binaries we can most accurately measure a combination of the component masses (the chirp mass) [54]. At the next level of accuracy, we can measure a combination of the binary's mass ratio and a combination of the parallel components of the black-hole masses, but not, at moderate signal-to-noise ratios, the individual black-hole spins [36,55-59]. To this picture we add the precession parameter $\chi_{p}$, which tells us that for the in-plane spin components, it is only one of them that we will most likely be able to measure.

How well we can measure each of these parameters, and what configurations allow us to sufficiently break the degeneracies in order to estimate both of the individual black-hole spins, will depend not only on the signal-tonoise ratio of the signal, but also the binary configuration and its relative orientation to the detector. This is a topic that deserves further study in the future, building on the work already done in Ref. [53].

Based on our results, we suggest that a waveform model with three spin parameters, which uses the same parameter 
reduction as in Sec. III, may be more effective in GW detection and parameter estimation than alternative parametrizations, for example the PTF reduction suggested in Ref. [37]. As we discussed in Sec. VI, this is because the parameter reduction we proposed accurately models separately the inspiral rate (using the two parallel spin components), as well as the dominant precession dynamics (using $\chi_{p}$ ). One could also consider a parameter reduction based on only two spin components, one for the effective parallel spin, and another for the effective precession spin. We find that these models perform well, with a significant improvement over the PTF mapping to single-spin systems.

The present study has been limited to inspiral waveforms only, and has not included match comparisons that are optimized over the source parameters; we have also neglected the effect of higher-PN-order spin terms, which may weaken the $\chi_{p}$ degeneracy that we have identified. The purpose here was to demonstrate the utility of a single precession parameter in capturing the average precession exhibited by a generic binary system. More detailed studies are required to determine the value of this parameter reduction in GW searches and in parameter estimation, and in waveforms that include merger and ringdown. Depending on the extent to which this partial degeneracy holds throughout the entire IMR waveform, it may be possible to accurately model generic binaries with NR simulations that cover a reduced parameter space, thus making far more tractable the problem of constructing generic IMR models for use in GW astronomy with advanced detectors. However, the identification of the dominant physical parameters in the inspiral is valuable in itself in simplifying the construction of precessing waveform models and in particular for producing a sufficient analytic description of the rotation that describes the evolution of the orbital plane; for example, the frequencydomain precessing IMR model proposed in Ref. [33] was motivated in part by a preliminary version of the results presented here.

\section{ACKNOWLEDGMENTS}

We thank G. Faye for providing us with a MATHEMATICA notebook containing all waveform-mode expressions used in this work. We also thank A. Bohé, S. Fairhurst, S. Husa, G. Pratten, M. Pürrer and B. Sathyaprakash for valuable discussions and for comments on the manuscript. P. S. was a recipient of a DOC-fFORTE-fellowship of the Austrian Academy of Sciences and was also partially supported by the STFC. M.H. was supported by a Science and Technology Facilities Council grant ST/H008438/1, and both M. H. and F. O. by ST/I001085/1.

\section{APPENDIX A: PN WAVEFORM GENERATION}

For efficiency reasons, the PN waveforms used in the analysis presented here are generated by integrating the orbit-averaged precession equations including leadingorder spin-orbit, spin-spin and radiation reaction contributions, under the assumption of a quasispherical inspiral for $\vec{L}$ and $\vec{S}_{i}$, as given in Ref. [26]:

$$
\begin{aligned}
\dot{\vec{L}}= & \frac{1}{r^{3}}\left[\left(2+\frac{3 q}{2}\right) \vec{S}_{1}+\left(2+\frac{3}{2 q}\right) \vec{S}_{2}\right] \times \vec{L} \\
& -\frac{3}{2 r^{3}}\left[\left(\vec{S}_{2} \cdot \hat{L}\right) \vec{S}_{1}+\left(\vec{S}_{1} \cdot \hat{L}\right) \vec{S}_{2}\right] \times \hat{L} \\
& -\frac{32 \mu^{2}}{5 r}\left(\frac{m}{r}\right)^{5 / 2} \hat{L}, \\
\dot{\vec{S}}_{1}=\frac{1}{r^{3}} & {\left[\left(2+\frac{3 q}{2}\right) \hat{L}+\frac{1}{2} \vec{S}_{2}-\frac{3}{2}\left(\vec{S}_{2} \cdot \hat{L}\right) \hat{L}\right] \times \vec{S}_{1}, } \\
\dot{\vec{S}}_{2}=\frac{1}{r^{3}} & {\left[\left(2+\frac{3}{2 q}\right) \hat{L}+\frac{1}{2} \vec{S}_{1}-\frac{3}{2}\left(\vec{S}_{1} \cdot \hat{L}\right) \hat{L}\right] \times \vec{S}_{2} . }
\end{aligned}
$$

The evolution equation for the precession angle $\alpha(t)$ is determined by differentiating Eq. (2.2) with respect to time,

$$
\dot{\alpha}(t)=\frac{L_{x} \dot{L}_{y}-L_{y} \dot{L}_{x}}{L_{x}^{2}+L_{y}^{2}} .
$$

The opening angle $t(t)$ is obtained from Eq. (2.1).

Further, we integrate the evolution equation for the orbital separation, $r$, and construct the orbital frequency, $\omega_{\text {orb }}$, from it:

$$
\begin{aligned}
\dot{r}(t)= & -\frac{64 \eta}{5}\left(\frac{m}{r}\right)^{3}\left[1-\frac{1}{336}(1751+588 \eta)-\left\{\frac{7}{12} \sum_{i=1,2}\left[\chi_{i}\left(\hat{L} \cdot \hat{S}_{i}\right)\left(19 \frac{m_{i}^{2}}{m^{2}}+15 \eta\right)\right]-4 \pi\right\}\left(\frac{m}{r}\right)^{3 / 2}\right. \\
& \left.-\frac{5}{48} \eta \chi_{1} \chi_{2}\left[59\left(\hat{S}_{1} \cdot \hat{S}_{2}\right)-173\left(\hat{L} \cdot \hat{S}_{1}\right)\left(\hat{L} \cdot \hat{S}_{2}\right)\right]\left(\frac{m}{r}\right)^{2}\right], \\
\omega_{\text {orb }}^{2}= & \left(\frac{m}{r^{3}}\right)\left\{1-(3-\eta)\left(\frac{m}{r}\right)-\sum_{i=1}^{2}\left[\chi_{i}\left(\hat{L} \cdot \hat{S}_{i}\right)\left(2 \frac{m_{i}^{2}}{m^{2}}+3 \eta\right)\right]\left(\frac{m}{r}\right)^{3 / 2}+\left[\left(6+\frac{41}{4} \eta+\eta^{2}\right)\right.\right. \\
& \left.\left.-\frac{3}{2} \eta \chi_{1} \chi_{2}\left[\left(\hat{S}_{1} \cdot \hat{S}_{2}\right)-3\left(\hat{L} \cdot \hat{S}_{1}\right)\left(\hat{L} \cdot \hat{S}_{2}\right)\right]\right]\left(\frac{m}{r}\right)^{2}\right\} .
\end{aligned}
$$


We then integrate the equation for the total phase, Eq. (2.4). The evolution is performed in the $J_{0}$-aligned frame and is terminated when a final separation of $r=6 \mathrm{M}$ (corresponding to the last stable circular orbit in the Schwarzschild spacetime) is reached. As initial conditions we choose the spin components defined with respect to $\hat{L}_{0} \equiv(0,0,1)$, the initial separation $r_{0}=40 M$, the initial orbital phase $\Phi_{0}=0$ and the initial azimuth of $\hat{L}$ in the $J_{0}$-aligned frame. We also have to set the initial magnitude of the orbital angular momentum, which we choose to be the Newtonian value, $L_{0} \equiv L_{N}=m_{1} m_{2} \sqrt{r_{0} / M}$. The transformation into the $J_{0}$-aligned frame is given by the following rotation matrix:

$$
\mathbf{R}=\mathbf{R}_{z}\left(\epsilon_{0}-\pi\right) \mathbf{R}_{y}\left(-l_{0}\right) \mathbf{R}_{z}\left(-\epsilon_{0}\right),
$$

where $\epsilon_{0}$ is the initial azimuth of the total angular momentum $J_{0}$.

Once we have solved for the dynamics of the binary, we use the mode expressions $h_{\ell m}$ as given in Ref. [60] to construct the precessing waveforms. We only use the $(\ell=2)$ modes and truncate the amplitudes at leading PN order $\left(v^{2}\right)$, yielding the following explicit mode expressions:

$$
\begin{gathered}
h_{22}=-\frac{A}{2} e^{-2 i(l-\alpha-\Phi)}\left[e^{4 i \phi}\left(-1+e^{i l}\right)^{4}+\left(1+e^{i l}\right)^{4}\right], \\
h_{21}=-i A e^{-i(\alpha+2 \Phi+2 l)}\left[-e^{4 i \Phi}\left(1+e^{i l}\right)\left(-1+e^{i l}\right)^{3}-\left(1+e^{i l}\right)^{3}\left(-1+e^{i l}\right)\right], \\
h_{20}=A \sqrt{\frac{3}{2}} e^{-2 i(l+\Phi)}\left(-1+e^{2 i l}\right)^{2}\left(1+e^{4 i \Phi}\right), \\
h_{2,-2}=-\frac{A}{2} e^{2 i(\alpha+\Phi+l)}\left[e^{-4 i(\Phi+\pi)}\left(-1+e^{-i l}\right)^{4}+\left(1+e^{-i l}\right)^{4}\right], \\
h_{2,-1}=i A e^{i(\alpha+2 l+2 \Phi+\pi)}\left[-e^{-4 i(\Phi+\pi)}\left(-1+e^{-i l}\right)^{3}\left(1+e^{-i l}\right)-\left(-1+e^{-i l}\right)\left(1+e^{-i l}\right)^{3}\right],
\end{gathered}
$$

where the amplitude factor is

$$
A=\frac{M \eta}{D_{L}} v^{2} \sqrt{\frac{\pi}{5}}
$$

In the above equations $D_{L}$ is the luminosity distance of the GW source which we set to $D_{L}=1$.

\section{APPENDIX B: GENERIC MATCHES}

Following the notation introduced in Sec. IV B, we define the real-valued detector response

$$
\begin{gathered}
h_{\text {resp }}(t)=\cos (2 \psi) h_{+}(t)+\sin (2 \psi) h_{\times}(t) \\
=\operatorname{Re}\left[h(t) e^{i 2 \psi}\right]
\end{gathered}
$$

where $\psi$ is the polarization angle and

$$
h=h_{+}-i h_{\times}
$$

is the complex GW strain. As discussed in Sec. IV B, we remind the reader that our definitions of $h_{+}$and $h_{\times}$include the orientation-dependent antenna pattern of the detector, except for the effect of a relative rotation in the detector plain that is explicitly governed by $\psi$.

Our goal is to formulate the inner product between a signal and a model response in terms of their complex GW strains. We first express the Fourier-domain detector response, $\tilde{h}_{\text {resp }}$, by

$$
\begin{gathered}
h_{\text {resp }}(t)=\frac{1}{2}\left[h(t) e^{i 2 \psi}+h^{*}(t) e^{-i 2 \psi}\right], \\
\Rightarrow \tilde{h}_{\text {resp }}(f)=\frac{1}{2}\left[\tilde{h}(f) e^{i 2 \psi}+\tilde{h}^{*}(-f) e^{-i 2 \psi}\right] .
\end{gathered}
$$

We then simply insert this expression into the inner product,

$$
\left\langle h_{\mathrm{resp}}^{S} \mid h_{\mathrm{resp}}^{M}\right\rangle=2 \int_{-\infty}^{\infty} \frac{\tilde{h}_{\mathrm{resp}}^{S}(f) \tilde{h}_{\mathrm{resp}}^{M *}(f)}{S_{n}(|f|)} d f,
$$

and group the terms conveniently

$$
\begin{aligned}
\left\langle h_{\text {resp }}^{S} \mid h_{\mathrm{resp}}^{M}\right\rangle= & \frac{1}{2} \int_{-\infty}^{\infty}\left[\tilde{h}^{S}(f) \tilde{h}^{M *}(f) e^{i 2\left(\psi_{S}-\psi_{M}\right)}+\tilde{h}^{S *}(-f) \tilde{h}^{M}(-f) e^{-i 2\left(\psi_{S}-\psi_{M}\right)}\right. \\
& \left.+\tilde{h}^{S}(f) \tilde{h}^{M}(-f) e^{i 2\left(\psi_{S}+\psi_{M}\right)}+\tilde{h}^{S *}(-f) \tilde{h}^{M *}(f) e^{-i 2\left(\psi_{S}+\psi_{M}\right)}\right] \frac{d f}{S_{n}(|f|)}
\end{aligned}
$$


Noting that

$$
\int_{-\infty}^{\infty} x(f) d f=\int_{-\infty}^{\infty} x(-f) d f
$$

for any integrable function $x$, we identify the first and last two terms in Eq. (B7) as complex conjugates of each other, respectively, which leads to the final expression

$$
\begin{aligned}
\left\langle h_{\mathrm{resp}}^{S} \mid h_{\mathrm{resp}}^{M}\right\rangle= & \operatorname{Re} \int_{-\infty}^{\infty} \frac{\tilde{h}^{S}(f) \tilde{h}^{M *}(f)}{S_{n}(|f|)} e^{2 i\left(\psi_{S}-\psi_{M}\right)} d f \\
& +\operatorname{Re} \int_{-\infty}^{\infty} \frac{\tilde{h}^{S}(f) \tilde{h}^{M}(-f)}{S_{n}(|f|)} e^{2 i\left(\psi_{S}+\psi_{M}\right)} d f .
\end{aligned}
$$

The first contribution in Eq. (B9) closely resembles the "standard" formulation of the inner product, where the overall phase difference is now identified as a difference of the polarization angles. The second term quantifies the asymmetry between positive and negative frequencies, or, equivalently, the nonstationarity in the waveform strain.

In the following we are interested in the normalized match between the signal and model, so we need to express the norm of each waveform which, according to Eq. (B9), reads

$$
\begin{aligned}
\left\|h_{\text {resp }}\right\|^{2} & =\left\langle h_{\text {resp }} \mid h_{\text {resp }}\right\rangle \\
& =\int_{-\infty}^{\infty} \frac{|\tilde{h}(f)|^{2}}{S_{n}(|f|)} d f+\operatorname{Re} \int_{-\infty}^{\infty} \frac{\tilde{h}(f) \tilde{h}(-f)}{S_{n}(|f|)} d f e^{4 i \mu} .
\end{aligned}
$$

Again, while the first term is similar to the standard norm of nonprecessing signals, there is a second (generally smaller) contribution that quantifies the asymmetry and makes the norm polarization dependent.

To find the optimal match over all polarization angles of the model, $\psi_{M}$, we rephrase the expressions above in terms of the real-valued quantities $N_{1}, N_{2}, O, \sigma_{N}$ and $\sigma_{O}$,

$$
\begin{aligned}
N_{1} & =\int_{-\infty}^{\infty} \frac{\left|\tilde{h}^{M}(f)\right|^{2}}{S_{n}(|f|)} d f, \\
N_{2} e^{i \sigma_{N}} & =\int_{-\infty}^{\infty} \frac{\tilde{h}^{M}(f) \tilde{h}^{M}(-f)}{S_{n}(|f|)} d f, \\
O e^{i \sigma_{O}} & =\int_{-\infty}^{\infty} \frac{\tilde{h}^{M *}(f)}{S_{n}(|f|)}\left[\tilde{h}^{S}(f) e^{2 i \psi_{S}}+\tilde{h}^{S *}(-f) e^{-2 i \psi_{S}}\right] d f .
\end{aligned}
$$

This allow us to express the optimized match in the following way:

$$
\begin{aligned}
\max _{\psi_{M}}\left\langle\frac{h_{\mathrm{resp}}^{S}}{\left\|h_{\mathrm{resp}}^{S}\right\|} \mid \frac{h_{\mathrm{resp}}^{M}}{\left\|h_{\mathrm{resp}}^{M}\right\|}\right\rangle & =\max _{\psi_{M}} \frac{O}{\left\|h_{\mathrm{resp}}^{S}\right\|} \frac{\cos \left(2 \psi_{M}-\sigma_{O}\right)}{\sqrt{N_{1}+N_{2} \cos \left(4 \psi_{M}+\sigma_{N}\right)}}=\frac{O}{\left\|h_{\mathrm{resp}}^{S}\right\|} \sqrt{\frac{N_{1}-N_{2} \cos \left(\sigma_{N}+2 \sigma_{O}\right)}{N_{1}^{2}-N_{2}^{2}}}, \\
\psi_{M}^{\mathrm{opt}} & =\frac{1}{2} \arctan \frac{N_{1} \sin \left(\sigma_{O}\right)+N_{2} \sin \left(\sigma_{N}+\sigma_{O}\right)}{N_{1} \cos \left(\sigma_{O}\right)-N_{2} \cos \left(\sigma_{N}+\sigma_{O}\right)} .
\end{aligned}
$$

Note that these expressions are understood as matches for constant signal parameters (including $\psi_{S}$ ). However, recomputing the matches for a range of signal polarizations $\psi_{S}$ is computationally cheap as only $O$ and $\sigma_{O}$ have to be reevaluated following Eq. (B11).

The other parameter that we optimize over is a relative time shift between the signal and the model, which enters the match (B9) as a complex modulation $e^{2 \pi i f \Delta t}$. As usual, we efficiently calculate the match for discretized time shifts via the inverse Fourier transform, which in our formulation only affects $O$ and $\sigma_{O}$.
Finally, we separate the model waveform into its five $\ell=2$ spherical harmonic modes and calculate the quantities in Eq. (B11) separately for each mode, which turns $N_{1}$ and $\mathrm{N}_{2}$ into complex matrices and $O$ into a vector of discrete inverse Fourier transforms. However, we only need to calculate those quantities once for a given set of intrinsic binary parameters and combine them appropriately for each set of orientation and polarization angles that we wish to analyze. 
[1] S. Waldman (LIGO Scientific Collaboration), arXiv:1103.2728.

[2] G. M. Harry (LIGO Scientific Collaboration), Classical Quantum Gravity 27, 084006 (2010).

[3] J. Aasi et al. (LIGO Scientific Collaboration, Virgo Collaboration), arXiv:1304.0670.

[4] Virgo Collaboration, Virgo Technical Report No. VIR-0027A-09, 2009, https://tds.ego-gw.it/itf/tds/file.php? callFile $=$ VIR-0027A-09.pdf.

[5] K. Somiya (LCGT Collaboration), Classical Quantum Gravity 29, 124007 (2012).

[6] L. Blanchet, Living Rev. Relativity 17, 2 (2014), http://www .livingreviews.org/lrr-2014-2.

[7] T. Damour, B. R. Iyer, and B. S. Sathyaprakash, Phys. Rev. D 57, 885 (1998).

[8] A. Buonanno and T. Damour, Phys. Rev. D 59, 084006 (1999).

[9] A. Buonanno and T. Damour, Phys. Rev. D 62, 064015 (2000).

[10] P. Ajith et al., Classical Quantum Gravity 24, S689 (2007).

[11] P. Ajith et al., Phys. Rev. D 77, 104017 (2008).

[12] A. Buonanno, Y. Pan, J. Baker, J. Centrella, B. Kelly, S. McWilliams, and J. van Meter, Phys. Rev. D 76, 104049 (2007).

[13] A. Buonanno, Y. Pan, H. Pfeiffer, M. Scheel, L. Buchman, and L. Kidder, Phys. Rev. D 79, 124028 (2009).

[14] T. Damour, A. Nagar, E. N. Dorband, D. Pollney, and L. Rezzolla, Phys. Rev. D 77, 084017 (2008).

[15] T. Damour, A. Nagar, M. Hannam, S. Husa, and B. Brügmann, Phys. Rev. D 78, 044039 (2008).

[16] T. Damour and A. Nagar, Phys. Rev. D 79, 081503 (2009).

[17] Y. Pan, A. Buonanno, M. Boyle, L. T. Buchman, L. E. Kidder, H. P. Pfeiffer, and M. A. Scheel, Phys. Rev. D 84, 124052 (2011).

[18] T. Damour, A. Nagar, and S. Bernuzzi, Phys. Rev. D 87, 084035 (2013).

[19] P. Ajith et al., Phys. Rev. Lett. 106, 241101 (2011).

[20] Y. Pan, A. Buonanno, L. T. Buchman, T. Chu, L. E. Kidder, H. P. Pfeiffer, and M. A. Scheel, Phys. Rev. D 81, 084041 (2010).

[21] L. Santamaria et al., Phys. Rev. D 82, 064016 (2010).

[22] A. Taracchini, Y. Pan, A. Buonanno, E. Barausse, M. Boyle, T. Chu, G. Lovelace, H. P. Pfeiffer, and M. A. Scheel, Phys. Rev. D 86, 024011 (2012).

[23] F. Ohme, Classical Quantum Gravity 29, 124002 (2012).

[24] A. Taracchini et al., Phys. Rev. D 89, 061502 (2014).

[25] T. A. Apostolatos, C. Cutler, G. J. Sussman, and K. S. Thorne, Phys. Rev. D 49, 6274 (1994).

[26] L. E. Kidder, Phys. Rev. D 52, 821 (1995).

[27] P. Schmidt, M. Hannam, S. Husa, and P. Ajith, Phys. Rev. D 84, 024046 (2011).

[28] M. Boyle, R. Owen, and H. P. Pfeiffer, Phys. Rev. D 84, 124011 (2011).

[29] R. O'Shaughnessy, J. Healy, L. London, Z. Meeks, and D. Shoemaker, Phys. Rev. D 85, 084003 (2012).

[30] P. Schmidt, M. Hannam, and S. Husa, Phys. Rev. D 86, 104063 (2012).
[31] L. Pekowsky, R. O'Shaughnessy, J. Healy, and D. Shoemaker, Phys. Rev. D 88, 024040 (2013).

[32] Y. Pan, A. Buonanno, A. Taracchini, L. E. Kidder, A. H. Mroué, H. P. Pfeiffer, M. A. Scheel, and B. Szilágyi, Phys. Rev. D 89, 084006 (2014).

[33] M. Hannam, P. Schmidt, A. Bohé, L. Haegel, S. Husa, F. Ohme, G. Pratten, and M. Pürrer, Phys. Rev. Lett. 113, 151101 (2014).

[34] P. Ajith, Phys. Rev. D 84, 084037 (2011).

[35] C. Reisswig, S. Husa, L. Rezzolla, E. Nils Dorband, D. Pollney, and J. Seiler, Phys. Rev. D 80, 124026 (2009).

[36] M. Pürrer, M. Hannam, P. Ajith, and S. Husa, Phys. Rev. D 88, 064007 (2013).

[37] A. Buonanno, Y. Chen, Y. Pan, and M. Vallisneri, Phys. Rev. D 70, 104003 (2004).

[38] A. Lundgren and R. O'Shaughnessy, Phys. Rev. D 89, 044021 (2014).

[39] M. Hannam, Gen. Relativ. Gravit. 46, 1767 (2014).

[40] T. A. Apostolatos, Phys. Rev. D 52, 605 (1995).

[41] T. A. Apostolatos, Phys. Rev. D 54, 2421 (1996).

[42] A. Buonanno, Y. Chen, and M. Vallisneri, Phys. Rev. D 67, 104025 (2003).

[43] C. Van Den Broeck, D. Brown, T. Cokelaer, I. Harry, G. Jones, B. Sathyaprakash, H. Tagoshi, and H. Takahashi, Phys. Rev. D 80, 024009 (2009).

[44] P. Jaranowski and A. Królak, Living Rev. Relativity 15, 4 (2012), http://www.livingreviews.org/lrr-2012-4.

[45] B. S. Sathyaprakash and B. F. Schutz, Living Rev. Relativity 12, 2 (2009), http://www.livingreviews.org/lrr-2009-2.

[46] B. Vaishnav, I. Hinder, F. Herrmann, and D. Shoemaker, Phys. Rev. D 76, 084020 (2007).

[47] S. T. McWilliams, B. J. Kelly, and J. G. Baker, Phys. Rev. D 82, 024014 (2010).

[48] L. Lindblom, B. J. Owen, and D. A. Brown, Phys. Rev. D 78, 124020 (2008).

[49] D. Shoemaker (LIGO Scientific Collaboration), https://dcc .ligo.org/LIGO-T0900288/public.

[50] I. W. Harry, A. H. Nitz, D. A. Brown, A. P. Lundgren, E. Ochsner, and D. Keppel, Phys. Rev. D 89, 024010 (2014).

[51] P. Ajith, N. Fotopoulos, S. Privitera, A. Neunzert, and A. Weinstein, Phys. Rev. D 89, 084041 (2014).

[52] D. A. Brown, A. Lundgren, and R. O'Shaughnessy, Phys. Rev. D 86, 064020 (2012).

[53] S. Vitale, R. Lynch, J. Veitch, V. Raymond, and R. Sturani, Phys. Rev. Lett. 112, 251101 (2014).

[54] K. G. Arun, B. R. Iyer, B. S. Sathyaprakash, and P. A. Sundararajan, Phys. Rev. D 71, 084008 (2005).

[55] C. Cutler and E. E. Flanagan, Phys. Rev. D 49, 2658 (1994).

[56] E. Poisson and C. M. Will, Phys. Rev. D 52, 848 (1995).

[57] E. Baird, S. Fairhurst, M. Hannam, and P. Murphy, Phys. Rev. D 87, 024035 (2013).

[58] M. Hannam, D. A. Brown, S. Fairhurst, C. L. Fryer, and I. W. Harry, Astrophys. J. 766, L14 (2013).

[59] F. Ohme, A. B. Nielsen, D. Keppel, and A. Lundgren, Phys. Rev. D 88, 042002 (2013).

[60] K. G. Arun, A. Buonanno, G. Faye, and E. Ochsner, Phys. Rev. D 79, 104023 (2009). 